\title{
Synthesis of new polyconjugated molecules with biphenyl, dibenzothiophene, carbazole and phenanthrene units
}

\author{
Vyacheslav K. Olkhovik,* Dmitrii A. Vasilevskii, Andrei A. Pap, Galina V. Kalechyts, \\ Yurii V. Matveienko, Andrei G. Baran, Nikolay A. Halinouski, and Vitalii G. Petushok \\ Institute of Chemistry of New Materials of National Academy of Sciences, F. Skorinii str., 36, \\ 220141, Minsk, Belarus \\ E-mail:slavol@ichnm.basnet.by
}

\section{Dedicated to Prof. Oleg Kulinkovich on the occasion of his 60th birthday}

\begin{abstract}
The simple methods of synthesis of hardly accessible substituted biphenyl, dibenzothiophene, carbazole and phenanthrene derivatives were elaborated starting from dimethyl 4,4'biphenyldicarboxylate. The series of new luminophores with extended $\pi$-conjugated chains based on combinations of biphenyl, carbazole, dibenzothiophene, phenanthrene fragments and alternating phenyl, vinyl or heterocyclic units were synthesized by the Wittig and the Knoevenagel reactions of corresponding aromatic dialdehydes and different $\mathrm{CH}$-acids or phosphonium salts. Investigation of the effect of various substituents on the luminescent properties has been presented. The new luminophores could be used as emissive or charge transport layers in organic light emitting diodes (OLEDs).
\end{abstract}

Keywords: Biphenyl, carbazole, dibenzothiophene, phenanthrene, conjugated system

\section{Introduction}

The biaryl axis is the central building block in a very large number of various molecules such as natural and pharmacologically active products, ${ }^{1}$ chiral reagents, ${ }^{2}$ as the source of chiral phases for chromatography, ${ }^{3}$ as inflexible "spacers" between two parts of a molecule, ${ }^{4,5}$ as the basis of liquid crystals ${ }^{6}$ and fluorescent layers in OLEDs. ${ }^{7}$ Different substituted biphenyls very often are used as suitable synthetic intermediates for synthesis heteroaromatic and polycyclic aromatic compounds. ${ }^{8}$ The key step in such synthesis almost always is the coupling of two matched aromatic parts of the target molecule. Another way is the directed modification of easily accessible biphenyls such as, for example, 4, 4'-biphenyldicarboxylic acid. 
Methyl ester of 4, 4'-biphenyldicarboxylic acid $\mathbf{1}$ is a very convenient and cheap starting material for synthesis of wide range of various biphenyl derivatives, especially because it can be separated from wastes of DMT manufacture. ${ }^{9}{ }^{10}$ Owing to presence of two para-carboxylic groups in this molecule, the conjugated system of biphenyl can be easily involved in construction of extensive polyconjugated molecules like $\mathbf{2}$, which are potential luminophores and electroactive materials. Additional fuctionalization of biphenyl rings allows varying properties of synthesized molecules. ${ }^{7,11-13}$

Similar heteroaromatic and polycyclic aromatic compounds 3-5 are of great interest as intermediates for synthesis of such kind polyconjugated systems. The analysis of literature shows that whereas of 2, 7-carbazolyl, 2,7-phenanthrenyl and 3,7-dibenzothiophenyl fragments have the lengthiest conjugation, they are not very often used as chromophores for preparation of dyes and luminophores. First of all, it is connected with difficulties of the direct functionalization of $\mathrm{C} 2$, $\mathrm{C} 3$ and $\mathrm{C} 7$ positions in these ring systems by simple electrophilic substitution. ${ }^{14-17}$

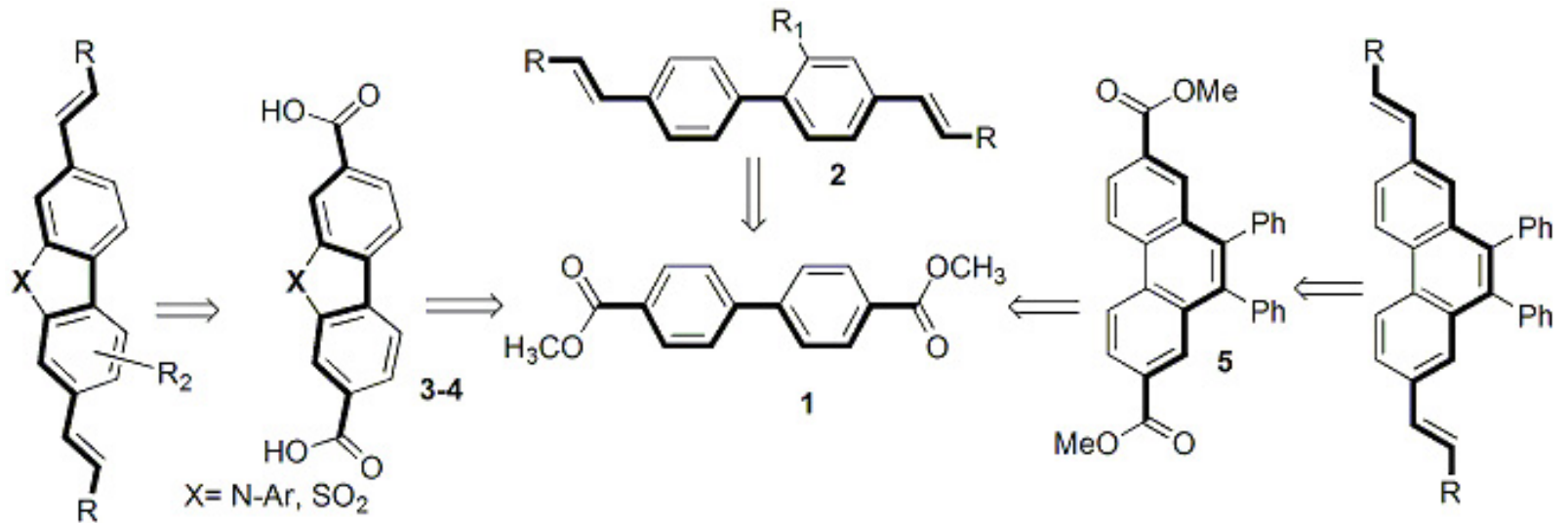

\section{Figure 1}

The object of this work was elaboration of effective methods of synthesis of difficult-toaccess 4,4'-disubstituted biphenyl, 3,7-disubstituted dibenzothiophene, 2,7-disubstituted carbazole and phenanthrene derivatives followed by their application in synthesis of polyconjugated systems - potential electroluminescent and electroactive materials (Figure 1).

\section{Results and Discussion}

There are at least two reasons to consider 4, 4'-biphenyldicarboxylic acid as a versatile starting material for synthesis of the above mentioned system. First, two carboxylic groups in this molecule are the very convenient functions to develop and to lengthen side chains. Second, the cross-effect of the para-carboxylic group and the second ring gives the only answer about the 
direction of electrophilic substitution in this molecule and allows the carrying out a necessary functionalization of the ring system.

The double sulphurization of diester 1 with chlorosulphonic acid gives 5, 5dioxodibenzothiophen-3, 7-dicarboxylic acid $\mathbf{3}$ in quantitative yield (Scheme 1). This compound itself is the suitable chromophoric building block for synthesis of the rod-shaped molecules with a lengthy $\pi$-conjugated system like 10, which are potential electroluminescent materials. The dioxazole derivative $\mathbf{1 0}$ was prepared in one step by refluxing of acid $\mathbf{3}$ with $\boldsymbol{o}$-aminophenol in the presence of boric acid ${ }^{10}$ in diphenyl ether.

The molecule of dibenzothiophene 3 contains three electron-withdrawing groups and we anticipated that the introduction of an additional electron-donating function in this molecule could dramatically affect its properties as a chromophor. Amino and hydroxy derivatives 7, 9 are the most interesting from the viewpoint of their further use in synthesis of luminescent materials. The key intermediate in the synthesis of both amino and hydroxy derivatives is the nitro dibenzothiophene 6 . The nitration of diacid 3 with nitric acid in concentrated $\mathrm{H}_{2} \mathrm{SO}_{4}$ proceeded with the highest regioselectivity giving 1-nitro-5,5-dioxodibenzothiophen 6 in $80 \%$ yield.

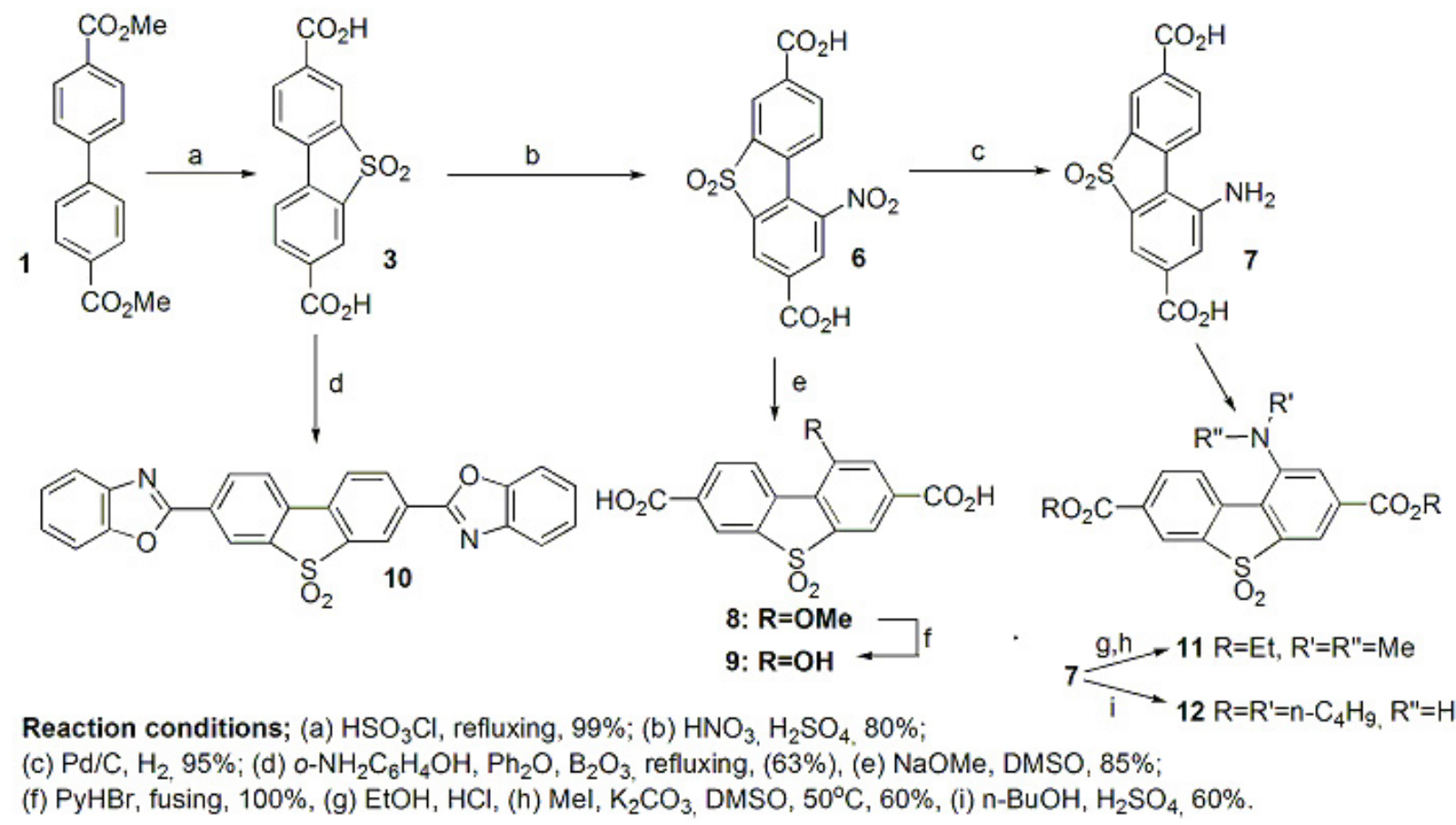

Scheme 1. Synthesis of 5, 5-dioxo-5H-dibenzo $[b, d]$ thiophene derivatives.

The minor 2-nitro derivative (5-7\%) was easily separated with thrice-repeated crystallization from isopropyl alcohol. The presence of two carboxylic and sulfo electron-withdrawing groups in the molecule makes possible the direct nucleophilic substitution of the nitro group in relatively mild conditions, and, therefore, 1-hydroxy derivative 9 was obtained by treatment of nitro 
compound 6 with $\mathrm{NaOMe}$ in DMSO at $100^{\circ} \mathrm{C}$ followed by fusion with pyridine hydrobromide. In contrast to the starting compound 3 1-hydroxy-5,5-dioxodibenzothiophen-3,7-dicarboxylic acid 9 intensively fluoresces in solutions and displays the strong red solvatochromic shift in emission spectra $\left(\lambda_{\text {fluor. }}=395\right.$ (toluene), $\lambda_{\text {fluor. }}=525$ (ethanol)).

1-Amino-5, 5-dioxodibenzothiophen-3,7-dicarboxylic acid 7 was obtained by palladium catalyzed hydrogenation of nitro derivative 6 in 95\% yield. Both the amino 7 and the alkylamino derivatives 11, 12 intensively fluoresce in the solid state and solutions in the blue-green region spectra $\left(\lambda_{\text {fluor. }}=470-510 \mathrm{~nm}\right)$ and show high thermal and light stability.

We used these chromophores for the synthesis of new polyconjugated systems, which comprised dibenzothiophene and stilbene structural elements. The simplest model compounds

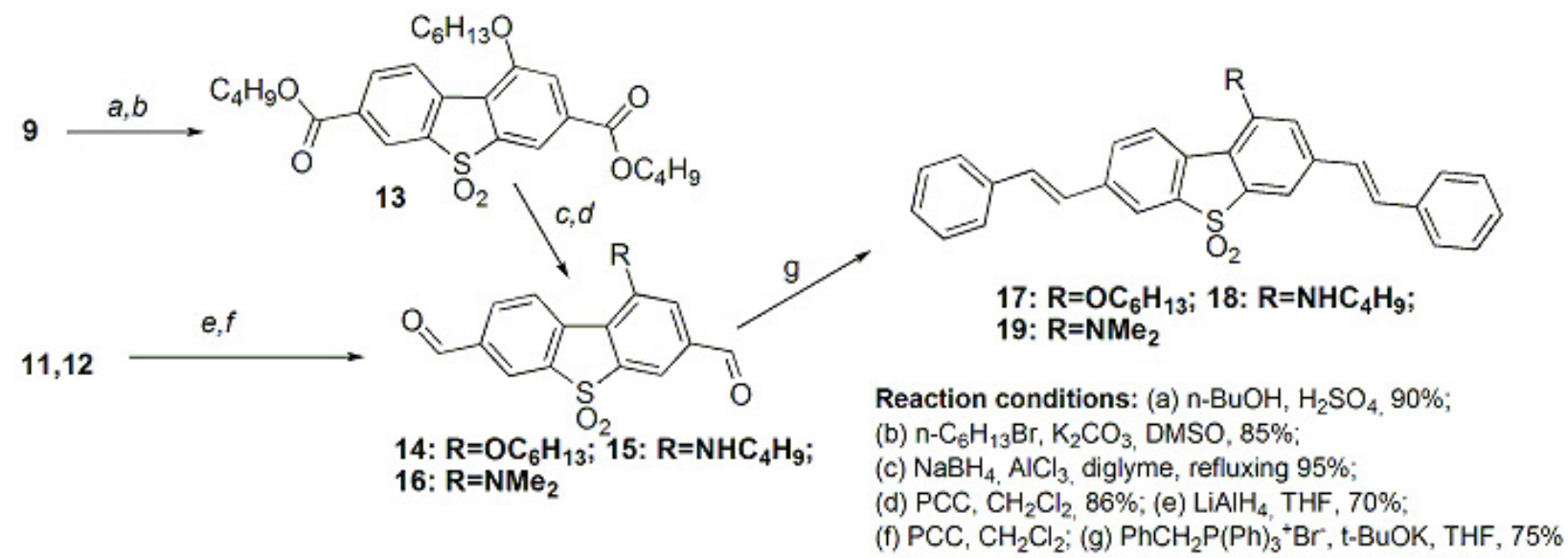

Scheme 2

17-19 with phenylenevinylene fragments in main chain were prepared by the Wittig reaction of aldehydes 14-16 with benzyltriphenylphosphonium bromide as depicted in the Scheme 2 .

The aldehydes were synthesized in two steps by the reduction of esters 11-13 followed by oxidation of corresponding alcohols with PCC. The ylide was generated under treatment of the phosphnium salt with $t$-BuOK in THF and the only trans-trans isomers of 17-19 were isolated in $45-75 \%$ yields. In comparison with the starting diesters, the highest effect was observed in the emission spectra of 2-alkoxy derivative 17. The red-shift of luminescence maximum was $50 \mathrm{~nm}$ ( $\lambda_{\text {fluor. }}=397$ (13), $\lambda_{\text {fluor. }}=448$ (17)) because of expanding of $\pi$-conjugated backbone. In the case of amines 18, 19 only as much as three times the increase of emission intensity was observed without any changes of maximums positions in PL spectra.

Prepared from 4,4'-biphenyldicarboxylic acid dibenzothiophenes $\mathbf{3 , 8}$ in their turn are very convenient intermediates to obtain 2- and 2,2'-subsituted 4,4'-biphenyldicarboxylic acids 20, 21, which could be used as starting materials for the synthesis of luminophores with conjugated extended chains of alternating phenyl, vinyl or heterocyclic units - potential electroluminescent (EL) materials (Scheme 3). 


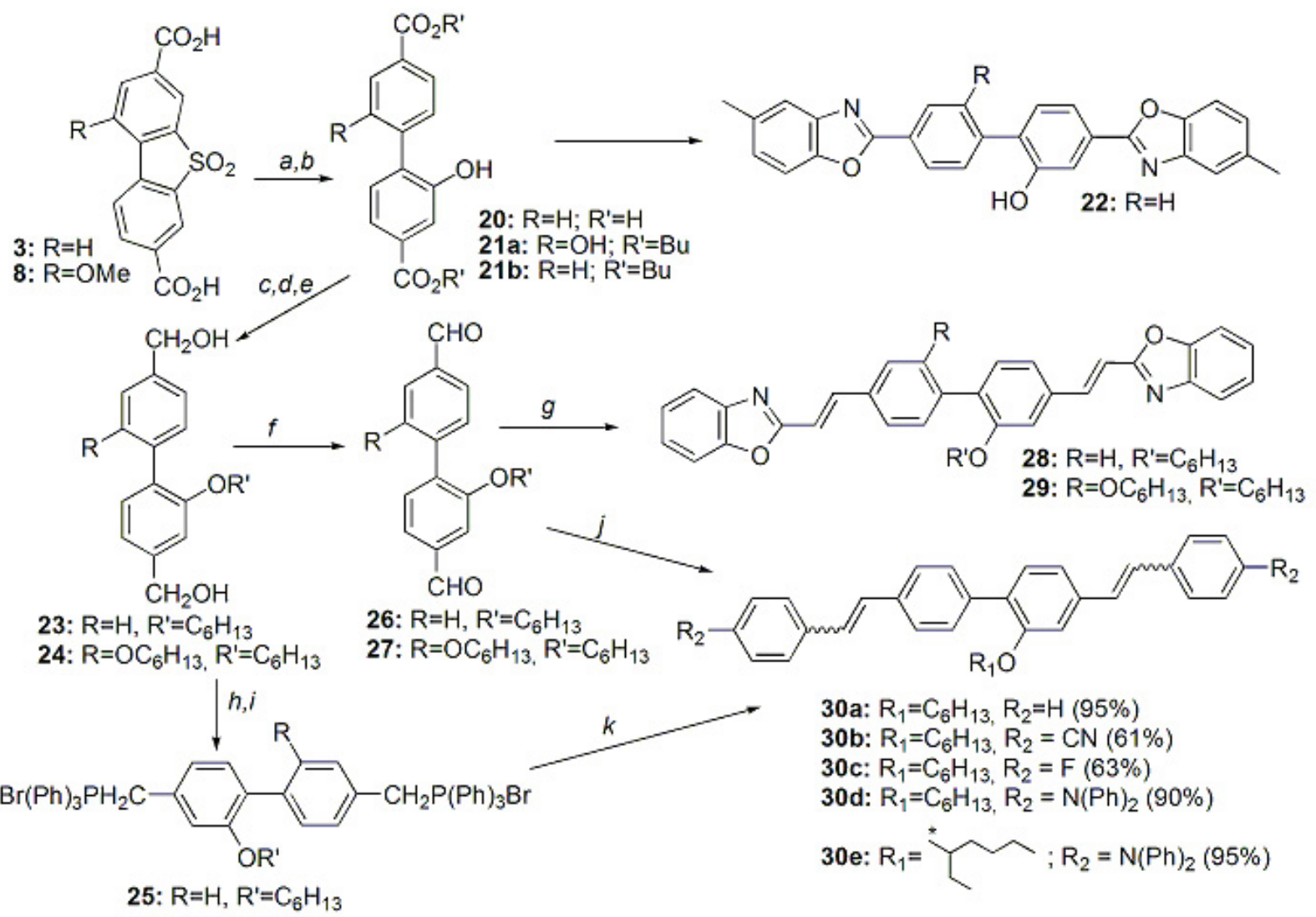

Reaction conditions: (a) $\mathrm{NaOH}$, alloyng, $97 \%$; (b) $\mathrm{BuOH}, \mathrm{H}_{2} \mathrm{SO}_{4}$; (c) $\mathrm{MeOH} / \mathrm{HCl}, 95 \%$; (d) $\mathrm{nC}_{6} \mathrm{H}_{13} \mathrm{Br}, \mathrm{K}_{2} \mathrm{CO}_{3}, \mathrm{DMSO}$, $80 \%$; (e) $\mathrm{LiAlH}_{4}$, THF, $90 \%$; (f) PCC, dioxane, 85\%; (g) NaOCH 3 , DMSO,2-methyl-1,3-benzoxazole, $80 \%$; (h) $\mathrm{PBr}_{3}, \mathrm{Et}_{2} \mathrm{O},-10^{\circ} \mathrm{C}$, $86 \%$; (i) $\mathrm{P}(\mathrm{Ph})_{3}$, DMF, $100^{\circ} \mathrm{C}$, $98 \%$; (j) t-BuOK, $\mathrm{C}_{6} \mathrm{H}_{5} \mathrm{CH}_{2} \mathrm{P}(\mathrm{Ph})_{3} \mathrm{Br}$, toluene, $55 \%$; (k) t-BuOK, toluene, $\mathrm{R}_{2}-\mathrm{C}_{6} \mathrm{H}_{4} \mathrm{CHO}$

Scheme 3. Synthesis of policonjugated molecules on basis of 2-substituted biphenyls.

Generally, luminophores from non-substituted biphenyls have low solubility and high crystallization tendency that impedes their application as EL materials, ${ }^{18,19}$ but owing to presence of hydroxy functions in compounds 28-30, one can correct their solubility and the glass transition temperature by introduction of long alkoxy groups.

2-Hydroxy-4, 4'-biphenyldicarboxylic acid $\mathbf{2 0}$ was obtained in high yield by simply fusing of sulphone 3 with $\mathrm{NaOH}$ at $250-280^{\circ} \mathrm{C}$. ${ }^{10}$ The same procedure with 2-methoxy sulphone 8 gave the mixture of 2- and 2, 2'-dihydroxy derivatives in a ratio 1:2 and 80\% total yield, and their dibutyl esters 21 a-b can be easy separated by extraction of monoester with petroleum ether in the Soxhlet extractor. The strong influence of the substituent at $\mathrm{C} 2$ position on luminescence was demonstrated by the example of bis-benzoxazolyl derivative 22, which was synthesized by condensation of diacid 20 with 4-methyl-2-aminophenole in mixture of diphenyl ether/pyridine and boric acid as catalyst. This compound shows intensive blue photoluminescence in DMSO solution $\left(\lambda_{\text {max. }}=420 \mathrm{~nm}\right)$ and, in the same time, luminescence of its salt form solution has maximum at $570 \mathrm{~nm}$ with the anomalous big Stokes shift $(\sim 240 \mathrm{~nm})$, when pair electrons of phenolate can be drawn into conjugated system. ${ }^{11}$ 
The synthesis of the rod-shaped molecules with ethenyl linkers between the biphenyl moiety and electron-withdrawing 1,3-oxazole group was fulfilled by the Knoevenagel reaction of 2substituted-4,4'-biphenyldicarbaldehydes $\mathbf{2 6}$ and $\mathbf{2 7}$ with 2-methyl-1,3-benzoxazole. The benzoxazole 28, due to its electron-rich and good thermal stability, was used as emissive layer to enhance organic light-emitting diodes (OLEDs). ${ }^{13}$

A series of new soluble compounds based on a combination of biphenyl and stilbene structural elements were synthesized by the Wittig reaction of the aldehyde 26 with benzyl (triphenyl)phosphonium salts or diphosphonium bromide 25 with various aromatic aldehydes. The dimethyl ester of $\mathbf{2 0}$ was reduced with $\mathrm{LiAlH}_{4}$ in THF and the resulting alcohol $\mathbf{2 3}$ was used for preparation both dialdehyde $\mathbf{2 6}$ and diphosphonium salt $\mathbf{2 5}$ as key intermediates in the synthesis of the EL dyes.

The stereochemistry of Wittig reaction products 30a-e depends very much on the structure of aromatic aldehydes. In case of relatively small molecules of benzaldehyde, 4-cyano- and 4fluorbenzaldehyde, the coupling of phosphorane generated from 25 does not proceed stereoselectively and inseparable mixtures of all four isomers (trans-trans, cis-cis, trans-cis) are formed. The same result was obtained when the aldehyde $\mathbf{2 6}$ reacted with benzyltriphenylphosphonium bromide. In both cases $t$-BuOK was used as base to generate corresponding ylides. The substances 30a-c show strong blue photoluminescence in solutions and, in spite of the fact that they are mixture of isomers, could be used as electroactive materials in manufacturing of OLEDs. Stereoselectivity of the reaction increased when we used the bulky 4-(diphenylamino)benzcarbaldehyde ${ }^{20}$ and the trans-trans isomer of 30d was separated in $75 \%$ yield. The rest, approx. $15 \%$, was a mixture of other isomers, which were completely converted in the more stable trans-trans isomer of $\mathbf{3 0 d}$ by the refluxing in toluene with catalytic amount of iodine under argon atmosphere. The diphosphonium bromide 25, where $\mathrm{R}$ ' is the branched 2ethyl-1-hexenyl, reacts with 4-(diphenylamino)benzcarbaldehyde giving the only trans-trans isomer 30e in 95\% yield. We anticipated that the luminophores like 30d,e with electron donating aromatic amines in the main chain of conjugation have to possess the balanced hole and electron injection abilities and could be promising EL materials.

The most commonly used the hole-transporting materials in organic light-emitting devices are triarylamines like 4, 4'-bis [N-(1-naphthyl-)- $N$-phenyl-amino]-biphenyl (NPB). ${ }^{21} \mathrm{~N}$-Arylated carbazole can be regarded as structural analog of triarylamines but having flat and rigid structure. That allows expecting good charge transporting properties, high termo- and light stability of polyconjugated molecules on their basis. ${ }^{22}$ One of the most common methods for synthesis of carbazole derivatives is the Cadogan reaction of 2-nitrobiphenyls in the presence of organophosphorus reagents. ${ }^{23}$ A requisite dimethyl 2-nitro [1, 1-biphenyl]-4,4'-dicarboxylate 31 was synthesized in high yield by nitration of diester 1 under thoroughly controlled temperature conditions. Unfortunately, the reductive intramolecular cyclization of $\mathbf{3 1}$ in the presence of triphenylphosphine gave only 30\% yield of desired dimethyl $9 H$-carbazole-2,7-dicarboxylate 32 (Scheme 4). 


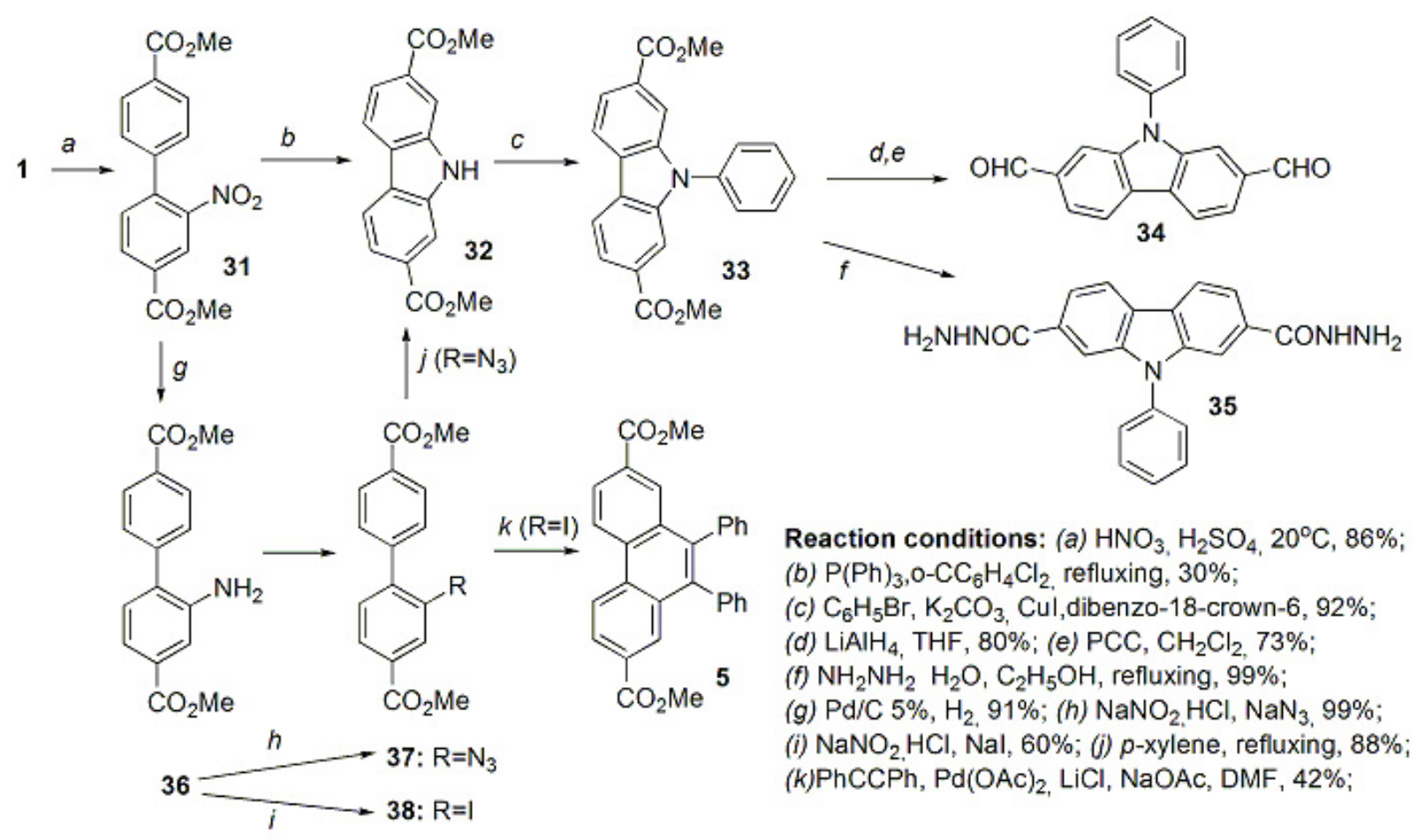

Scheme 4. Synthesis of carbazole and phenanthrene derivatives.

Furthermore, the reaction mixture was contaminated by a number of byproducts that were diddicult to separate. Another way for generation of singlet nitrenes that easily react with introduction into aromatic C-H bond is thermo- or photocyclization of 2-azidobiphenyls. The chain of transformations involved the reduction of nitro derivative $\mathbf{3 1}$ in amine 36, formation diazonium salt, its substitution with $\mathrm{NaN}_{3}$ and thermocyclyzation of the azide 37. These transformations gave the carbazole 32 in $80 \%$ total yield, starting from nitrobiphenyl 31. The arylation of the electron-deficient nitrogen atom in dimethyl carbazole-2,7-dicarboxylate $\mathbf{3 2}$ demands harsh reaction conditions, however, the refluxing of it with bromobenzene in the presence of $\mathrm{K}_{2} \mathrm{CO}_{3}, \mathrm{CuI}$ and dibenzo-18-crown- 6 gave the desired $\mathrm{N}$-aryl carbazole 33 in unexpectedly high yield (92\%). The corresponding dialdehyde $\mathbf{3 4}$ was obtained in two steps as depicted in Scheme 4. The carbazole-2, 7-dicarbohydrazide 35 was synthesized in quantitative yield by refluxing with hydrazine hydrate in ethanol.

Palladium-catalyzed annulation of aryl iodides with alkynes has been proved to be a useful method of forming polycyclic aromatic compounds, in particular substituted phenanthrenes. ${ }^{24,25}$ The crucial 2-iodobiphenyl $\mathbf{3 8}$ was obtained by nucleophilic substitution of the amino group in 36 via the corresponding diazonium salt. To synthesize 9, 10-diphenylphenanthrene 5 we used an annulation procedure described for monosubstituted 2-iodobiphenyls. ${ }^{25}$

Both the carbazoles 34, 35 and phenanthrene 5 are versatile building blocks for synthesis of lengthy $\pi$-conjugated systems with alternating electron donor and electron acceptor groups in the main chain of conjugation, which could show good balanced luminescence and charge transport 
properties. The electron withdrawing ability of the 1, 3, 4-oxadiazole and 1,3-oxazole groups are very strong and comparable to the nitrile one. The presence of double bonds in these heterocycles allows using them as chromophore elements and in the same time as linkers for extending of a conjugated system. The synthesis of the carbazole derivative $\mathbf{3 9}$ with benzoxazole units in the side chain was accomplished by the condensation of the dialdehyde 34 with 2-methyl1,3-benzoxazole under the Knoevenagel reaction conditions (Scheme 5). The oligomer 40, in which carbazole moiety is connected with bromophenyl unit via 1,3,4-oxadiazole linkers, was synthesized in $42 \%$ total yield by reaction of the dihydrazide 35 with 4-bromobenzoyl chloride followed by cyclization of the corresponding dibenzoyl carbazole-2,7-dicarbohydrazide in boiling
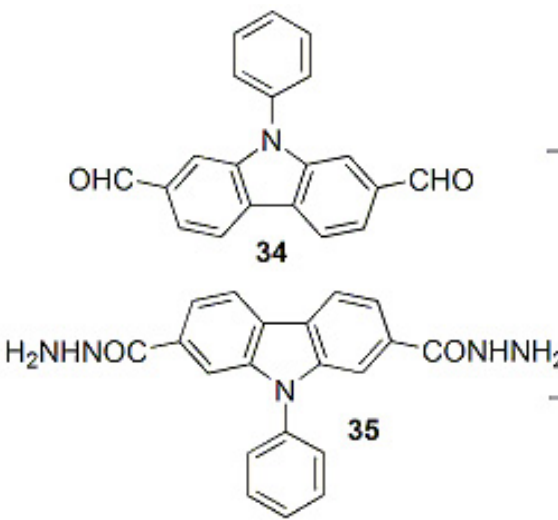

$b, c$

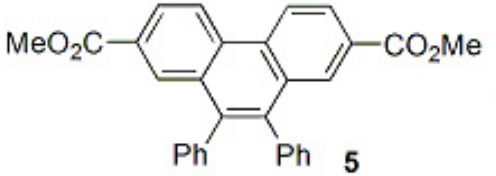

Reaction conditions: (a) t-BuOK, DMSO,2-methyl-1,3-benzoxazole, $78 \%$; (b) 4- $\mathrm{BrC}_{6} \mathrm{H}_{4} \mathrm{COCl}$, $\mathrm{Py}$; (c) $\mathrm{POCl}_{3}$, refluxing $46 \%$; (d) $\mathrm{NaOH}, \mathrm{EtOH} / \mathrm{THF}$; (e) $\mathrm{SOCl}_{2}$, DMF; (f) p- $\mathrm{C}_{8} \mathrm{H}_{17} \mathrm{OC}_{6} \mathrm{H}_{4} \mathrm{CONHNH}_{2}$, THF, Et ${ }_{3} \mathrm{~N}$; (g) POCl $\mathrm{PO}_{3}$, refluxing $66 \%$

\section{Scheme 5}

$\mathrm{POCl}_{3}$. The dibromide 40 itself intensively luminesces in the blue region $\left(\lambda_{\max }=428.5 \mathrm{~nm}\right)$ and could be used in synthesis of polymeric EL materials by the Suzuki-Miyaura cross coupling with boric acids. ${ }^{26}$ The invert succession of transformations was realized for preparation of phenanthrene 41 because of poor solubility of the diester 5 and the corresponding dihydrazide. 9,10-Diphenyl-2,7-phenanthrenedicarbonyl dichloride was synthesized in two steps, and without further purification was condensed with 4-(octyloxy)benzenecarbohydrazide to give the mixed dihydrazide, which was cyclized in compound $\mathbf{4 1}$ in high total yield. This dye has strong blue fluorescence and good solubility in common organic solvents. 


\section{Experimental Section}

General Procedures. All melting points were measured using a Boetius apparatus and are uncorrected. IR spectra were recorded using a UR-20 IR spectrometer. ${ }^{1} \mathrm{H}$ NMR (100 or 500, 13 $\mathrm{MHz})$ and ${ }^{13} \mathrm{C}$ NMR $(125,75 \mathrm{MHz})$ spectra were recorded as $\mathrm{CDCl}_{3}$ and DMSO-d $\mathrm{d}_{6}$ solutions on a Tesla BS-567A and Bruker AVANCE-500 instruments respectively. Chemical shifts $(\delta)$ are given from TMS $(0 \mathrm{ppm})$ as internal standard for ${ }^{1} \mathrm{H}-\mathrm{NMR}$, and ${ }^{13} \mathrm{CDCl}_{3}(77.0 \mathrm{ppm})$ for ${ }^{13} \mathrm{C}-$ NMR. TLC was performed on aluminum backed silica gel 60 F254 plates and visualized by UV and/or exposure to $I_{2}$. Column chromatography was conducted with Merck Kieselgel 60: 70-230 mesh. Solvents were dried and freshly distilled according to common practice.

5, 5-Dioxo-5H-dibenzo[b,d]thiophene-3,7-dicarboxylic acid (3). A solution of $27 \mathrm{~g}(0.1 \mathrm{~mol})$ dimethyl ester of 4,4'-biphenyldicarboxylic acid in $116.5 \mathrm{~g}(1 \mathrm{~mol})$ chlorosulfonic acid was heated at reflux for $3 \mathrm{~h}$ (cease of evolution of $\mathrm{HCl}$ ). The reaction mixture was cooled and poured on $1 \mathrm{~kg}$ of crushed ice. The precipitated crystals were filtered off, washed with water up to neutral medium and dried. The yield was $30.21 \mathrm{~g}(99 \%)$. MP $>400^{\circ} \mathrm{C}$ (dec.); Elemental Anal. Calcd for $\mathrm{C}_{14} \mathrm{H}_{8} \mathrm{O}_{6} \mathrm{~S}$ : C 55.26, H 2.65, S 10.54 Found: C 55.01, H 2.53, S 10.36; ${ }^{1} \mathrm{H}$ NMR (DMSO-d $6,100 \mathrm{MHz}$ ), $\delta: 4.5-6.2$ (br s, 2H, $-\mathrm{OH}), 8.3$ (s, 6H, Ar); IR (KBr, $v \mathrm{~cm}^{-1}$ ): 3430, 2630, $2530,1710,1670,1620,1450,1420,1310,1260,1180,1150,1090,950,860,770,710$.

Dimethyl 9, 10-diphenylphenanthrene-2,7-dicarboxylate (5). A mixture of dimethyl 2iodobiphenyl-4, 4'-dicarboxylate (38) $(1.3 \mathrm{~g}, 3.2 \mathrm{mmol})$, LiCl $(0.138 \mathrm{~g}, 3.2 \mathrm{mmol}), 1,2-$ diphenylethyne $(0.65 \mathrm{~g}, 3.5 \mathrm{mmol})$, palladium diacetate $(0.04 \mathrm{~g}, 5 \mathrm{~mol} \%)$ and sodium acetate 0.5 $\mathrm{g}, 6.4 \mathrm{mmol})$ in $50 \mathrm{ml}$ of DMFA was stirred at $95-105^{\circ} \mathrm{C}$ for 3 days. After cooling, the reaction mixture was poured into $100 \mathrm{ml}$ of water and the precipitate was filtered off and washed with hexane. After drying, $0.61 \mathrm{~g}(42 \%)$ of 5 as a yellow powder was obtained. $\mathrm{MP}=309-310^{\circ} \mathrm{C}$; Elemental Anal. Calcd for $\mathrm{C}_{30} \mathrm{H}_{22} \mathrm{O}_{4}$ : C 80.70, H 4.97 Found: C 80.47, H 4.88; ${ }^{1} \mathrm{H}$ NMR $\left(\mathrm{CDCl}_{3}\right.$, $500 \mathrm{MHz}), \delta: 3.81\left(\mathrm{~s}, 6 \mathrm{H},-\mathrm{CH}_{3}\right), 7.2-7.4(\mathrm{~m}, 10 \mathrm{H},-\mathrm{Ph}), 8.09(\mathrm{~s}, 2 \mathrm{H}, 1-, 8-\mathrm{H}), 8.22\left(\mathrm{~d}, 2 \mathrm{H}, \mathrm{J}^{\text {ortho }}\right.$ $=9 \mathrm{~Hz}, 3-, 6-\mathrm{H}), 9.13\left(\mathrm{~d}, 2 \mathrm{H}, \mathrm{J}^{\text {ortho }}=9 \mathrm{~Hz}, 3-, 6-\mathrm{H}\right) . \mathrm{IR}\left(\mathrm{KBr}, v_{\mathrm{cm}}{ }^{-1}\right): 3100,3050,2970,1730$, $1570,1440,1390,1340,1300,1260,1210,1130,1020,970,830,770,710$.

1-Nitro-5, 5-dioxo-5H-dibenzo $[\boldsymbol{b}, \boldsymbol{d}]$ thiophene-3,7-dicarboxylic acid (6). To a solution of $2 \mathrm{~g}$ (0.0065 mol) 5, 5'-dioxodibenzothiophen-3, 7-dicarboxylic acid (3) in $60 \mathrm{ml}$ of $\mathrm{H}_{2} \mathrm{SO}_{4}, 0.64 \mathrm{ml}$ $(0.013 \mathrm{~mol}) \mathrm{HNO}_{3}(70 \%)$ was added dropwise at $80^{\circ} \mathrm{C}$. The resulting reaction mixture was stirred for $40 \mathrm{~min}$, then was cooled and diluted with $100 \mathrm{ml}$ of water. The precipitate was filtered off and washed with water up to neutral medium. Recrystallization from toluene gave white crystals. Yield: $2.07 \mathrm{~g}(90 \%), \mathrm{MP}=335-337^{\circ} \mathrm{C}$; Elemental Anal. Calcd for $\mathrm{C}_{14} \mathrm{H}_{7} \mathrm{NO}_{8} \mathrm{~S}: \mathrm{C} 48.14$, H 2.02, N 4.01, S 9.18 Found: C 47.80, H 1.89, N 3.99, S 9.09; ${ }^{1} \mathrm{H}$ NMR (DMSO-d 6 , 100 MHz), $\delta: 7.98\left(\mathrm{dd}, 1 \mathrm{H}, \mathrm{J}^{\text {ortho }}=8.4 \mathrm{~Hz}, \mathrm{~J}^{\text {para }}=0.5 \mathrm{~Hz}, 9-\mathrm{H}\right), 8,38\left(\mathrm{dd}, 1 \mathrm{H}, \mathrm{J}^{\text {ortho }}=8.4 \mathrm{~Hz}, \mathrm{~J}^{\text {meta }}=1.6 \mathrm{~Hz}\right.$, 8-H), $8.54\left(\mathrm{dd}, 1 \mathrm{H}, \mathrm{J}^{\mathrm{meta}}=1.6 \mathrm{~Hz}, \mathrm{~J}^{\text {para }}=0.5 \mathrm{~Hz}, 6-\mathrm{H}\right), 8.72\left(\mathrm{~d}, 1 \mathrm{H}, \mathrm{J}^{\text {meta }}=1.6 \mathrm{~Hz}, 4-\mathrm{H}\right), 8.74(\mathrm{~d}$, $\left.1 \mathrm{H}, \mathrm{J}^{\mathrm{meta}}=1.6 \mathrm{~Hz}, 2-\mathrm{H}\right)$; IR $\left(\mathrm{KBr}, v \mathrm{~cm}^{-1}\right): 3090,2630,1730,1610,1540,1400,1370,1315$, $1230,1170,1155,1060,940,920,910,865,810,775,755,745,700,650,575,535,520$. 
1-Amino-5,5-dioxo-5H-dibenzo[b,d]thiophene-3,7-dicarboxylic acid (7). To a solution of $5 \mathrm{~g}$ $(0.015 \mathrm{~mol})$ nitro derivative (6) in $100 \mathrm{ml}$ of THF, $0.5 \mathrm{~g} 5 \% \mathrm{Pd} / \mathrm{C}$ was added and the resulting mixture was placed in hydrogenating apparatus and stirred at $60^{\circ} \mathrm{C}$ under $10 \mathrm{~atm}$. pressure of hydrogen for $48 \mathrm{~h}$. The catalyst was filtered off and washed with THF. Solvent was removed in vacuum, the residue was recrystallized from toluene to give yellow crystals. Yield: $3.29 \mathrm{~g} \mathrm{(70 \% ).}$ $\mathrm{MP}=360^{\circ} \mathrm{C}$; Elemental Anal. Calcd for $\mathrm{C}_{14} \mathrm{H}_{9} \mathrm{NO}_{6} \mathrm{~S}$ : C 52.66, H 2.84, N 4.39, S 10.04 Found: $\mathrm{C}$ 52.60, H 2.73, N 4.28, S 9.87; ${ }^{1} \mathrm{H}$ NMR (DMSO-d $6,100 \mathrm{MHz}$ ), $\delta: 7.9$ (dd, $1 \mathrm{H}, \mathrm{J}^{\text {ortho }}=8.4 \mathrm{~Hz}$, $\left.\mathrm{J}^{\text {meta }}=1.4 \mathrm{~Hz}, 8-\mathrm{H}\right), 8.3-8.5(\mathrm{~m}, 4 \mathrm{H})$; IR $\left(\mathrm{KBr}, v_{\mathrm{cm}}{ }^{-1}\right): 3450,3300,1725,1640,1610,1560$, 1430, 1410, 1310, 1275, 1190, 1120, 1040, 1015, 980, 900, 875, 865, 825, 805, 760, 700, 635.

1-Methoxy-5, 5-dioxo-5H-dibenzo [b,d]thiophene-3,7-dicarboxylic acid (8). To a solution of 1-nitro-5, 5-dioxodibenzothiophen-3, 7-dicarboxylic acid 6 (3 g, $10 \mathrm{mmol})$ in $50 \mathrm{ml}$ of DMSO, $1.56 \mathrm{~g}(0,029 \mathrm{~mol}) \mathrm{MeONa}$ was added. The reaction mixture was heated at $110^{\circ} \mathrm{C}$ and was stirred for $2 \mathrm{~h}$. Then the reaction mixture was cooled, the formed crystals were filtered off, washed with acetone and then solved in water. The solution was acidified with $10 \%$ hydrochloric acid The precipitated crystals were separated by filtration and washed with water. Recrystallization from ethanol gave white crystals. Yield: $2.14 \mathrm{~g}(75 \%)$. MP $=360-361{ }^{\circ} \mathrm{C}$. Elemental Anal. Calcd for $\mathrm{C}_{15} \mathrm{H}_{10} \mathrm{O}_{7} \mathrm{~S}$ : C 53.89, H 3.02, S 9.59 Found: C 53.50, H 2.91, S 9.42; ${ }^{1} \mathrm{H}$ NMR (DMSO-d $\left.6,500 \mathrm{MHz}\right), \delta: 4.13\left(\mathrm{~s}, 3 \mathrm{H},-\mathrm{OCH}_{3}\right), 7,87$ (d, 1H, J ${ }^{\text {meta }}=1 \mathrm{~Hz}, 2-$ or 4-H), $7.93\left(\mathrm{~d}, 1 \mathrm{H}, \mathrm{J}^{\mathrm{meta}}=1 \mathrm{~Hz}, 2-\right.$ or 4-H), 8.2-8.5 (m, $3 \mathrm{H}, 6-$, 8-, 9-H); IR (KBr, v cm $\left.{ }^{-1}\right): 3460,2985$, 2640, 2590, 1840,1705, 1615, 1500, 1480, 1440, 1425, 1410, 1320, 1265, 1185, 1155, 1145 , 1060, 1020, 950, 925, 870, 760, 730, 710, 655.

1-Hydroxy-5, 5-dioxo-5H-dibenzo $[b, d]$ thiophen-3,7-dicarboxylic acid (9). A mixture composed of $5 \mathrm{~g}(0.012 \mathrm{mmol})$ methyl ether 8 and $20 \mathrm{~g}(0.17 \mathrm{mmol})$ pyridine hydrochloride was heated at $220^{\circ} \mathrm{C}$ and the resulting fusion was held at this temperature for $30 \mathrm{~min}$. Then the mixture was cooled down and diluted with $50 \mathrm{ml}$ of water. Precipitated crystals were filtered off and washed with water. Recrystallization from ethanol gave white crystals. Yield: $4.2 \mathrm{~g} \mathrm{(87.5 \% ).}$ $\mathrm{MP}>380^{\circ} \mathrm{C}$ (subl.); Elemental Anal. Calcd for $\mathrm{C}_{14} \mathrm{H}_{8} \mathrm{O}_{7} \mathrm{~S}$ : C 52.50, H 2.52, S 10.01 Found: C 52.27, H 2.41, S 9.88; ${ }^{1} \mathrm{H}$ NMR (DMSO-d 6 , $\left.500 \mathrm{MHz}\right), \delta: 7.79$ (d, 1H, J ${ }^{\text {meta }}=1 \mathrm{~Hz}, 2-$ or 4-H), $7.81\left(\mathrm{~d}, 1 \mathrm{H}, \mathrm{J}^{\text {meta }}=1 \mathrm{~Hz}, 2-\right.$ or $\left.4-\mathrm{H}\right), 8.33(\mathrm{~m}, 1 \mathrm{H}, 6-\mathrm{H}), 8.34\left(\mathrm{dd}, 1 \mathrm{H}, \mathrm{J}^{\text {ortho }}=7 \mathrm{~Hz}, \mathrm{~J}^{\text {meta }}=1.5 \mathrm{~Hz}\right.$, $8-\mathrm{H}), 8.50\left(\mathrm{dd}, 1 \mathrm{H}, \mathrm{J}^{\text {ortho }}=7 \mathrm{~Hz}, \mathrm{~J}^{\mathrm{para}}=0.7 \mathrm{~Hz}, 9-\mathrm{H}\right) . \mathrm{IR}\left(\mathrm{KBr}, \mathrm{cm}^{-1}\right): 3465,3100,1735,1595$, $1610,1435,1410,1310,1265,1195,1070,985,920,880,780,765,720,690,650$.

3, 7-Bis [5-methylbenzoxazol-2-yl]-5, 5-dioxo-5H-dibenzo [b,d]thiophene (10). A reaction mixture composed of $3 \mathrm{~g}(0.01 \mathrm{~mol})$ 5,5'-dioxodibenzothiophen-3,7-dicarboxylic acid 3, $2 \mathrm{~g}$ (0.02 mol) 2-amino-4-methylphenol, $0.2 \mathrm{~g}(0,003 \mathrm{~mol}) \mathrm{H}_{3} \mathrm{BO}_{3}, 50 \mathrm{ml}$ diphenyl ether and $10 \mathrm{~g}$ $(0.13 \mathrm{~mol})$ pyridine was heated at $180^{\circ} \mathrm{C}$ with stirring under argon atmosphere for $2 \mathrm{~h}$. Then the temperature was raised up to $220^{\circ} \mathrm{C}$ and the reaction mixture was stirred for additional $3 \mathrm{~h}$ with slow distillation of pyridine and nascent water. The reaction mixture was cooled at RT and precipitated crystals were filtered off and washed successively with toluene and acetone. Recrystallization from toluene gave yellow crystals. Yield $2.7 \mathrm{~g}(63 \%)$. MP $>250^{\circ} \mathrm{C}$ (dec.); Elemental Anal. Calcd for $\mathrm{C}_{28} \mathrm{H}_{18} \mathrm{~N}_{2} \mathrm{O}_{4} \mathrm{~S}$ : C 70.28, H 3.79, N 5.85, S 6.70 Found: C 69.85, H 
3.67, N 5.76, S 6.59; ${ }^{1} \mathrm{H}$ NMR (DMSO-d 6 , $\left.500 \mathrm{MHz}\right), \delta: 2.31\left(\mathrm{~s}, 6 \mathrm{H},-\mathrm{CH}_{3}\right), 7.01\left(\mathrm{~d}, \mathrm{~J}^{\text {ortho }}=8.0\right.$ $\mathrm{Hz}, 2 \mathrm{H}, 6^{\prime}, 6^{\prime}$ '/7', 7''-benzooxazolyl), 7.14 (d, J ortho $=8.0 \mathrm{~Hz}, 2 \mathrm{H}, 6$ 6',6'"/7',7' '-benzooxazolyl), 7.76 (s, 2H, 4',4' '-benzooxazolyl), 8.43 (d, J ${ }^{\text {ortho }}=7.0 \mathrm{~Hz}, 2 \mathrm{H}, 2,8 / 1,9-\mathrm{H}$ thioph.), 8.47 (d, J ${ }^{\text {ortho }}$ $=7.0 \mathrm{~Hz}, 2 \mathrm{H}, 2,8 / 1,9-\mathrm{H}$ thioph.), 8,63 (s, $2 \mathrm{H}, 4,6-\mathrm{H}$ thioph.); IR (KBr, $\left.v \mathrm{~cm}^{-1}\right)$ : 3100, 3047, 2997, 2963, 2893, 1750, 1687, 1667, 1623, 1580, 1567, 1545, 1497, 1480, 1430, 1380, 1367, 1342, 1306, 1290, 1277, 1232, 1206, 1180, 1168, 1116, 1100, 1043, 1023, 980, 932, 874, 845, 829, 800, 760; UV (DMF, $\left.\lambda_{\max }, \mathrm{nm}\left(\varepsilon, 1 /\left(\mathrm{mol}^{*} \mathrm{~cm}\right)\right)\right): 368.5$ (19600); FLU (DMF, $\lambda_{\max }, \mathrm{nm}, \lambda_{\mathrm{EX}}=$ $365.0 \mathrm{~nm}) 443.5$.

$N, \quad N$-Dimethyl-1-amino-5,5-dioxo-5H-dibenzo $[b, d]$ thiophen-3,7-dicarboxylic acid diethyl ether (11). 1-Amino-5,5-dioxodibenzothiophen-3,7-dicarboxylic acid 7 (3.3 g, $10 \mathrm{mmol})$ was dissolved in $60 \mathrm{ml}$ of ethanol saturated with $\mathrm{HCl}$, then the reaction mixture was heated at reflux and stirred for $12 \mathrm{~h}$. After cooling, a precipitate was filtered off and successively washed with ethanol and water. Dried crystals were solved in $40 \mathrm{ml}$ of DMSO, 2,5 g (18.7 mmol) $\mathrm{K}_{2} \mathrm{CO}_{3}$ and $2,5 \mathrm{~g}(17.7 \mathrm{mmol}) \mathrm{CH}_{3} \mathrm{I}$ were added, and then the reaction mixture was heated at $60^{\circ} \mathrm{C}$ and stirred for $140 \mathrm{~h}$. The reaction mixture was diluted with water and extracted with $\mathrm{CH}_{3} \mathrm{Cl}$. Combined organic solution was dried over $\mathrm{Na}_{2} \mathrm{SO}_{4}$. Concentration of the solution in vacuum afforded a residue, which was purified by column chromatography on silica gel (toluene) to give $1.9 \mathrm{~g}(60 \%)$ of $\mathbf{1 1}$ as a green solid. Recrystallization from ethanol afforded green needles with $\mathrm{mp}=210-212^{\circ} \mathrm{C}$. Elemental Anal. Calcd for $\mathrm{C}_{20} \mathrm{H}_{21} \mathrm{NO}_{6} \mathrm{~S}$ : C 59.54, H 5.25, N 3.47, S 7.95 Found: C 59.18, H 5.13, N 3.41, S 7.82; ${ }^{1} \mathrm{H}$ NMR $\left(\mathrm{CDCl}_{3}, 500 \mathrm{MHz}\right), \delta: 0.87(\mathrm{t}, 6 \mathrm{H}, \mathrm{J}=7 \mathrm{~Hz}$, $\left.\left(\mathrm{CH}_{3}\right)_{2}\right), 2.88\left(\mathrm{~s}, 6 \mathrm{H}, \mathrm{N}\left(\mathrm{CH}_{3}\right)_{2}\right), 4.41-4.45\left(\mathrm{~m}, 4 \mathrm{H},-\mathrm{CH}_{2}-\right), 8.06(\mathrm{~s}, 1 \mathrm{H}, 2-\mathrm{H}), 8.17(\mathrm{~s}, 1 \mathrm{H}, 4-\mathrm{H})$, $8.35\left(\mathrm{~d}, 1 \mathrm{H}, \mathrm{J}^{\text {ortho }}=8 \mathrm{~Hz}, 9-\mathrm{H}\right), 8.45\left(\mathrm{~d}, 1 \mathrm{H}, \mathrm{J}^{\text {ortho }}=8,5 \mathrm{~Hz}, 8-\mathrm{H}\right), 8.50(\mathrm{~s}, 1 \mathrm{H}, 6-\mathrm{H})$; IR $(\mathrm{KBr}, \mathrm{v}$ $\left.\mathrm{cm}^{-1}\right)$ : 3435, 3000, 2960, 2805, 1720, 1605, 1540, 1450, 1395, 1370, 1345, 1305, 1255, 1165, $1115,1025,985,905,860,830,805,770,755,720,685$.

$\mathrm{N}$-Butyl-1-amino-5, 5-dioxo-5H-dibenzo $[\boldsymbol{b}, d]$ thiophene-3,7-dicarboxylic acid dibutyl ether (12). A solution of $2.4 \mathrm{~g}(0.75 \mathrm{mmol})$ amine (7) and $0.5 \mathrm{ml} \mathrm{H}_{2} \mathrm{SO}_{4}$ in $30 \mathrm{ml}$ of butanol was heated $7 \mathrm{~h}$ with regular distillation of nascent water. The reaction mixture was cooled, diluted with $\mathrm{CHCl}_{3}$ and washed with $100 \mathrm{ml}$ of $5 \% \mathrm{NaHCO}_{3}$ and water. Concentration of the solution in vacuum afforded a residue, which was purified by column chromatography on silica gel (toluene) to give $2 \mathrm{~g} \mathrm{(55 \% )}$ of $\mathbf{1 2}$ as a green solid. Recrystallization from toluene afforded green crystals with $\mathrm{mp}=171-172^{\circ} \mathrm{C}$. Elemental Anal. Calcd for $\mathrm{C}_{26} \mathrm{H}_{33} \mathrm{NO}_{6} \mathrm{~S}: \mathrm{C} 64.04, \mathrm{H}$ 6.82, N 2.87, S 6.58 Found: C 63.76, H 6.69, N 2.75, S 6.49; ${ }^{1} \mathrm{H}$ NMR $\left(500 \mathrm{MHz}, \mathrm{CDCl}_{3}\right), \delta$ : $-1.06(\mathrm{~m}$, $\left.9 \mathrm{H}, \mathrm{CH}_{3}-\right), 1.45-1.60\left(\mathrm{~m}, 6 \mathrm{H},-\mathrm{CH}_{2}-\right), 1.75-1.82\left(\mathrm{~m}, 6 \mathrm{H},-\mathrm{CH}_{2}-\right), 3.33-3.36\left(\mathrm{~m}, 2 \mathrm{H},-\mathrm{NH}-\mathrm{CH}_{2}-\right.$ ), 4.34-4.40 (m, 4H, - $\left.\mathrm{OCH}_{2}-\right), 4.40(\mathrm{~s}, 1 \mathrm{H},-\mathrm{NH}-), 7.26(\mathrm{~s}, 1 \mathrm{H}, 2-\mathrm{H}), 7.84\left(\mathrm{~d}, 1 \mathrm{H}, \mathrm{J}^{\mathrm{meta}}=1,5 \mathrm{~Hz}, 4-\right.$ $\mathrm{H}), 7.87\left(\mathrm{~d}, 1 \mathrm{H}, \mathrm{J}^{\text {ortho }}=8 \mathrm{~Hz}, 9-\mathrm{H}\right), 8.36\left(\mathrm{dd}, 1 \mathrm{H}, \mathrm{J}^{\text {meta }}=1.5 \mathrm{~Hz}, \mathrm{~J}^{\text {ortho }}=8 \mathrm{~Hz}, 8-\mathrm{H}\right), 8.46(\mathrm{~d}, 1 \mathrm{H}$, $\left.\mathrm{J}^{\text {meta }}=1.5 \mathrm{~Hz}, 6-\mathrm{H}\right) ; \mathrm{IR}\left(\mathrm{KBr}, v \mathrm{~cm}^{-1}\right): 2975,2950,2890,1730,1610,1585,1490,1395,1305$, $1255,1180,1155,1130,1070,1035,950,900,860,775,750,640,600$.

1-Hexyloxy-5, 5-dioxo-5H-dibenzo [b,d] thiophen-3,7-dicarboxylic acid dibutyl ether (13). A mixture composed of 1-hydroxy-5,5-dioxodibenzothiophen-3,7-dicarboxylic acid (9) (2.2 g, 7 mmol), $30 \mathrm{ml}$ butanol and $0.5 \mathrm{ml} \mathrm{H}_{2} \mathrm{SO}_{4}$ was boiled for $10 \mathrm{~h}$ with regular distillation of nascent 
water. Then the reaction mixture was cooled, diluted with toluene and washed with $100 \mathrm{ml}$ of $5 \%$ solution of $\mathrm{NaHCO}_{3}$. The organic phase was dried over sodium sulfate and solvent was removed in vacuum. The residue without additional purification was solved in $40 \mathrm{ml}$ of DMSO, $2,5 \mathrm{~g}$ $(18.9 \mathrm{mmol})$ of $\mathrm{K}_{2} \mathrm{CO}_{3}$ and 2, $0 \mathrm{~g}(12.1 \mathrm{mmol})$ of 1-bromhexane were added. The reaction mixture was heated at $60^{\circ} \mathrm{C}$ and stirred for $12 \mathrm{~h}$. After cooling-off, the mixture was diluted with water and was extracted with chloroform. Combined organic phases were dried over $\mathrm{Na}_{2} \mathrm{SO}_{4}$. Concentration of the solution in vacuum afforded a residue, which was purified by recrystallization from toluene to give $\mathbf{1 3}(3 \mathrm{~g}, 85 \%)$ as white crystals. $\mathrm{MP}=80-82^{\circ} \mathrm{C}$; Elemental Anal. Calcd for $\mathrm{C}_{28} \mathrm{H}_{36} \mathrm{O}_{7} \mathrm{~S}$ : C 65.09, H 7.02, S 6.21 Found: C 64.73, H 6.86, S 6.08; ${ }^{1} \mathrm{H}$ NMR (DMSO-d 6 , $500 \mathrm{MHz}$ ), $\delta: 0.94$ (t, 3H, J = $7 \mathrm{~Hz}-\mathrm{C}_{3}$ ), 1.01 (t, 6H, J=7 Hz, - $\underline{\mathrm{C}}_{3}$ ), 1.36-1.60 (m, $\left.12 \mathrm{H},-\mathrm{CH}_{2}-\right), 1.77\left(\mathrm{~m}, 4 \mathrm{H},-\left(\mathrm{CH}_{2}\right)_{2}-\right), 1.83\left(\mathrm{~m}, 2 \mathrm{H},-\mathrm{CH}_{2}-\right), 4.28\left(\mathrm{t}, 2 \mathrm{H}, \mathrm{J}=6.5 \mathrm{~Hz}, \mathrm{Hex}-\mathrm{OCH}_{2}-\right)$, $4.38\left(\mathrm{t}, 4 \mathrm{H}, \mathrm{J}=6.5, \mathrm{~Hz}, \mathrm{Bu}-\mathrm{OCH}_{2^{-}}\right), 7.85\left(\mathrm{~d}, 1 \mathrm{H}, \mathrm{J}^{\text {meta }}=1 \mathrm{~Hz}, 2-\mathrm{H}\right), 8,09\left(\mathrm{~d}, 1 \mathrm{H}, \mathrm{J}^{\text {meta }}=1 \mathrm{~Hz}, 4-\right.$ $\mathrm{H}), 8.33\left(\mathrm{~m}, 1 \mathrm{H}, \mathrm{J}^{\text {ortho }}=8.5 \mathrm{~Hz}, \mathrm{~J}^{\text {meta }}=1.5 \mathrm{~Hz}, 8-\mathrm{H}\right), 8.42\left(\mathrm{~d}, 1 \mathrm{H}, \mathrm{J}^{\text {ortho }}=8.5 \mathrm{~Hz}, 9-\mathrm{H}\right), 8.48(\mathrm{~d}$, $\left.1 \mathrm{H}, \mathrm{J}^{\mathrm{meta}}=1.5 \mathrm{~Hz}, 6-\mathrm{H}\right)$; IR $\left(\mathrm{KBr}, v \mathrm{~cm}^{-1}\right): 2985,2640,2590,1840,1705,1615,1500,1480$, $1440,1425,1410,1320,1265,1185,1155,1145,1060,1020,950,925,870,760,730,710,655$.

1-Hexyloxy-5, 5-dioxo-5H-dibenzo [b,d]thiophene-3,7-dicarbaldehyde (14). To a stirred solution of $11.6 \mathrm{~g}(0.3 \mathrm{~mol}) \mathrm{NaBH}_{4}$ in $60 \mathrm{ml}$ of diglyme, $3.8 \mathrm{~g}(0.02 \mathrm{~mol})$ of $\mathrm{AlCl}_{3}$ was added and, after $15 \mathrm{~min}, 4.5 \mathrm{~g}(0.009 \mathrm{~mol})$ of dibutyl ether $\mathbf{1 3}$ was added. The resulting reaction mixture was heated at $110^{\circ} \mathrm{C}$ and was intensively stirred for $6 \mathrm{~h}$, then was cooled at RT and quenched with $100 \mathrm{ml}$ of water. Precipitated crystals were filtered off and washed with water. The crude product was dissolved in $30 \mathrm{ml}$ of $\mathrm{CHCl}_{3}, 3 \mathrm{~g}$ PCC was added and the reaction mixture was stirred at RT for $10 \mathrm{~h}$. Then $5 \mathrm{ml}$ of isopropanol was added to quench excess of PCC, the resulting solution was filtered through a layer of silica gel, the filtrate was concentrated in vacuum and the residue was purified by column chromatography to give $\mathbf{1 4}(2.8 \mathrm{~g}, 86 \%)$ as a white solid. Recrystallization from toluene gives white crystals. $\mathrm{MP}=142-144^{\circ} \mathrm{C}$; Elemental Anal. Calcd for $\mathrm{C}_{20} \mathrm{H}_{20} \mathrm{O}_{5} \mathrm{~S}$ : C 64.50, H 5.41, S 8.61 Found: C 64.12, H 5.28, S 8.25; ${ }^{1} \mathrm{H}$ NMR (DMSO-d $6,500 \mathrm{MHz}), \delta: 0.89\left(\mathrm{t}, 3 \mathrm{H}, \mathrm{J}=7 \mathrm{~Hz}, \mathrm{CH}_{3}\right), 1.30-1.39\left(\mathrm{~m}, 4 \mathrm{H},\left(\mathrm{CH}_{2}\right)_{2}\right), 1.53(\mathrm{~m}, 2 \mathrm{H}$, $\left.\mathrm{CH}_{2}\right), 1.93\left(\mathrm{~m}, 2 \mathrm{H}, \mathrm{CH}_{2}\right), 4.39\left(\mathrm{t}, 2 \mathrm{H}, \mathrm{J}=6,5 \mathrm{~Hz},-\mathrm{OCH}_{2}-\right), 7.96(\mathrm{~s}, 1 \mathrm{H}, 2-\mathrm{H}), 8.12\left(\mathrm{~d}, 1 \mathrm{H}, \mathrm{J}^{\text {meta }}=\right.$ $1 \mathrm{~Hz}, 4-\mathrm{H}), 8.33\left(\mathrm{~m}, 1 \mathrm{H}, \mathrm{J}^{\text {ortho }}=8.5 \mathrm{~Hz}, \mathrm{~J}^{\text {meta }}=1.5 \mathrm{~Hz}, 8-\mathrm{H}\right), 8.54(\mathrm{~s}, 1 \mathrm{H}, 6-\mathrm{H}), 8.56\left(\mathrm{~d}, 1 \mathrm{H}, \mathrm{J}^{\text {ortho }}\right.$ $=8.5 \mathrm{~Hz}, 9-\mathrm{H}), 10.09(\mathrm{~s}, 1 \mathrm{H},-\mathrm{CHO}), 10.10(\mathrm{~s}, 1 \mathrm{H},-\mathrm{CHO}) ; \mathrm{IR}\left(\mathrm{KBr}, v \mathrm{~cm}^{-1}\right): 3450,3080,2975$, 2950, 2875, 1700, 1610, 1570, 1490, 1440, 1400, 1350, 1325, 1290, 1215, 1160, 1050, 920, 875, $860,725,626$.

$\mathrm{N}$-Butyl-1-amino-5, 5-dioxo-5H-dibenzo $\quad[\boldsymbol{b}, \boldsymbol{d}]$ thiophen-3,7-dicarbaldehyde (15). To a suspension of $0.37 \mathrm{~g}(10 \mathrm{mmol}) \mathrm{LiAlH}_{4}$ in $50 \mathrm{ml}$ of THF, $3.0 \mathrm{~g}(6 \mathrm{mmol})$ of N-butyl-1-amino-5, 5-dioxodibenzothiophen-3,7-dicarboxylic acid dibutyl ether 12 in $30 \mathrm{ml}$ of THF was added. The reaction mixture was stirred for $12 \mathrm{~h}$, excess of $\mathrm{LiAlH}_{4}$ was quenched with $1 \mathrm{ml}$ of $5 \% \mathrm{NaOH}$, and then an inorganic precipitate was filtered off and washed with THF. The filtrate was dried over sodium sulfate and concentration of the solution in vacuum afforded $1.6 \mathrm{~g}$ (74\%) of 1butylamino-3, 7-bis (hydroxymethyl)-5H-dibenzo[b,d]thiophene-5,5-dione as a green solid, which was recrystallized from ethanol. $\mathrm{MP}=210-212^{\circ} \mathrm{C}$. Elemental Anal. Calcd. for 
$\mathrm{C}_{18} \mathrm{H}_{21} \mathrm{NO}_{4} \mathrm{~S}$ : C 62.23, H 6.09, N 4.03, S 9.23 Found: C 61.89, H 6.00, N 3.96, S 9.07; ${ }^{1} \mathrm{H}$ NMR $\left(\mathrm{DMSO}_{\mathrm{d}}, 500 \mathrm{MHz}\right), \delta: 0.87\left(\mathrm{t}, 3 \mathrm{H}, \mathrm{J}=8 \mathrm{~Hz}, \mathrm{CH}_{3}\right), 1.41\left(\mathrm{~m}, 2 \mathrm{H}, \mathrm{CH}_{2}\right), 1.64\left(\mathrm{~m}, 2 \mathrm{H}, \mathrm{CH}_{2}\right)$, $3.23\left(\mathrm{~m}, 2 \mathrm{H}, \mathrm{N}-\mathrm{CH}_{2}\right), 4.54\left(\mathrm{t}, 2 \mathrm{H}, \mathrm{J}=6 \mathrm{~Hz}, \mathrm{CH}_{2}-\mathrm{O}\right), 4.59$ (t, 2H, J = 6 Hz, CH $\left.\mathrm{CH}_{2} \mathrm{O}\right), 5.42$ (t, 1H, J $=6 \mathrm{~Hz}, \mathrm{OH}), 5.48(\mathrm{t}, 1 \mathrm{H}, \mathrm{J}=6 \mathrm{~Hz}, \mathrm{OH}), 5.89(\mathrm{t}, \mathrm{J}=5,5 \mathrm{~Hz}, \mathrm{NH}), 6.99(\mathrm{~s}, 1 \mathrm{H}, 2-\mathrm{H}), 7.06(\mathrm{~d}, 1 \mathrm{H}$, $\left.\mathrm{J}^{\text {meta }}=0.5 \mathrm{~Hz}, 4-\mathrm{H}\right), 7.65\left(\mathrm{dd}, 1 \mathrm{H}, \mathrm{J}^{\text {ortho }}=8 \mathrm{~Hz}, \mathrm{~J}^{\text {meta }}=1,5 \mathrm{~Hz}, 8-\mathrm{H}\right), 7.79\left(\mathrm{~d}, 1 \mathrm{H}, \mathrm{J}^{\text {meta }}=1,5 \mathrm{~Hz}\right.$, 6-H), $8.12(\mathrm{~d}, 1 \mathrm{H}, \mathrm{J}=8.5 \mathrm{~Hz}, 9-\mathrm{H})$; IR $\left(\mathrm{KBr}, v \mathrm{~cm}^{-1}\right): 3400,2975,2950,2880,1610,1570,1450$, $1410,1285,1210,1150,1065,1010,930,890,820,720,625$. The oxidation of the obtained bis(hydroxymethyl) derivative ( $1 \mathrm{~g}, 2 \mathrm{mmol})$ with PCC (2 $\mathrm{g})$ in $20 \mathrm{ml}$ of $\mathrm{CHCl}_{3}$ according to the procedure for $\mathbf{3 4}$ gave $0.6 \mathrm{~g}$ of $\mathbf{1 5}$ as a orange solid, which was used in next stage without purification.

1-Dimethylamino-5, 5-dioxo-5Hdibenzo $\quad[b, d]$ thiophene-3,7-dicarbaldehyde (16). The dialdehyde $16(0.3 \mathrm{~g})$ was obtained as an orange solid according to the procedure for 15 from $2 \mathrm{~g}$ $(4 \mathrm{mmol})$ of diester 11. The crude product was used in the next stage without any purification.

1-Hexyloxy-3, 7-bis [(E)-2-phenylethenyl]-5H-dibenzo $[b, d]$ thiophene-5,5-dione (17). To a stirred suspension of triphenylbenzylphosphonium bromide $(0.5 \mathrm{~g}, 1,1 \mathrm{mmol})$ in $40 \mathrm{ml}$ of dry THF, $t$-BuOK $(0.12 \mathrm{~g}, 1 \mathrm{mmol})$ was added. The reaction mixture was stirred approx. for $15 \mathrm{~min}$ until a homogeneous dark-red solution formed. Then a solution of $\mathbf{1 4}$ (185 mg, $0.5 \mathrm{mmol})$ in 10 $\mathrm{ml}$ of THF was added dropwise and stirring was continued for $40 \mathrm{~min}$. Concentration of reaction mixture in vacuum afforded a residue, which was purified by column chromatography on silica gel (toluene) to give $194 \mathrm{mg}(\mathbf{7 5 \%})$ of $\mathbf{1 7}$ as a yellow solid. Recrystallization from toluene afforded yellow crystals. $\mathrm{MP}=172-173^{\circ} \mathrm{C}$; Elemental Anal. Calcd for $\mathrm{C}_{34} \mathrm{H}_{32} \mathrm{O}_{3} \mathrm{~S}$ : C 78.43, $\mathrm{H}$ 6.19, S 6.16 Found: C 78.71, H 5.58, S 6.42; ${ }^{1} \mathrm{H}$ NMR (DMSO-d 6 , $500 \mathrm{MHz}$ ), $\delta: 0.90$ (t, 3H, J = $\left.7.5 \mathrm{~Hz}, \mathrm{CH}_{3}\right), 0.91-1.57\left(\mathrm{~m}, 4 \mathrm{H},\left(\mathrm{CH}_{2}\right)_{2}\right), 1.53\left(\mathrm{~m}, 2 \mathrm{H}, \mathrm{CH}_{2}\right), 1.94\left(\mathrm{~m}, 2 \mathrm{H}, \mathrm{CH}_{2}\right), 4.33(\mathrm{t}, 2 \mathrm{H}, \mathrm{J}=$ $\left.6.5 \mathrm{~Hz},-\mathrm{OCH}_{2}\right), 7.41-7.30(\mathrm{~m}, 8 \mathrm{H}$, arom), 7.55-7.84 (m, 7H, arom ), 7.84 (s, 1H, 4-H thioph.), $7.96\left(\mathrm{dd}, 1 \mathrm{H}, \mathrm{J}^{\text {ortho }}=8 \mathrm{~Hz}, \mathrm{~J}^{\text {meta }}=1.5 \mathrm{~Hz}, 8-\mathrm{H}\right.$ thioph. $), 8.21$ (d, $1 \mathrm{H}, \mathrm{J}^{\text {ortho }}=8 \mathrm{~Hz}, 9-\mathrm{H}$ thioph.), 8.26 (d, $1 \mathrm{H}, \mathrm{J}^{\text {meta }}=1.5 \mathrm{~Hz}, 6-\mathrm{H}$ thioph.); ${ }^{13} \mathrm{C}$ NMR (DMSO-d6, 125.75 MHz), $\delta: 14.11,22.68$, 25.81, 26.89, 29.12, 31.49, 31.87, 69.01, 111.12, 114.63, 118.84, 126.07, 126.46, 126.58, 126.80, $126.86,128.35,128.49,128.82,131.08,131.60,132.05,136.29,136.47,141.26,156.20$; IR $\left(\mathrm{KBr}, v \mathrm{~cm}^{-1}\right): 3040,2950,2870,1630,1590,1500,1450,1415,1300,1160,995,820,845,790$, 700, 620; FLU (toluene, $\lambda_{\max }, \mathrm{nm}, \lambda_{\mathrm{EX}}=383.0 \mathrm{~nm}$ ) 448.0 .

1-Butylamino-3, 7-bis $[(E)-2-p h e n y l e t h e n y l]-5 H$-dibenzo $\quad[b, d]$ thiophene-5,5-dione (18). According to the procedure for obtaining of 17: triphenylbenzylphosphonium bromide (1.8 g, 4 $\mathrm{mmol}), t$-BuOK $(0.45 \mathrm{~g}, 4 \mathrm{mmol})$, the crude product $15(0.6 \mathrm{~g})$. After purification 18 (486 $\mathrm{mg}$, $20 \%$ calculated from diester 12) was obtained as a yellow solid. $\mathrm{MP}=262-264^{\circ} \mathrm{C}$ (toluene). Elemental Anal. Calcd for $\mathrm{C}_{32} \mathrm{H}_{29} \mathrm{NO}_{2} \mathrm{~S}$ : C 78.18, H 5.95, N 2.85, S 6.52 Found: C 78.42, H 5.79, N 2.74, S 6.72; ${ }^{1} \mathrm{H}$ NMR (DMSO-d 6 , $500 \mathrm{MHz}$ ), $\delta: 0.99$ (t, 3H, J = 7.5 Hz, CH ), 1.48 (m, 2H, $\left.\mathrm{CH}_{2}\right), 1.71\left(\mathrm{~m}, 2 \mathrm{H}, \mathrm{CH}_{2}\right), 3.33\left(\mathrm{~m}, 2 \mathrm{H}, \mathrm{N}-\mathrm{CH}_{2}\right), 5.99(\mathrm{t}, 1 \mathrm{H}, \mathrm{J}=5.5,-\mathrm{NH}), 7.20(\mathrm{~s}, 1 \mathrm{H}, 2-\mathrm{H}$ thioph.), 7.30-7.43 (m, 8H, arom.), $7.56(\mathrm{~d}, 2 \mathrm{H}, \mathrm{J}=16.5 \mathrm{~Hz}$, trans- $\mathbf{C H}=\mathrm{CH}), 7.55-7.67(\mathrm{~m}, 5 \mathrm{H}$, arom.), $7.92\left(\mathrm{~m}, 1 \mathrm{H}, \mathrm{J}^{\text {ortho }}=8 \mathrm{~Hz}, \mathrm{~J}^{\text {meta }}=1 \mathrm{~Hz}, 8-\mathrm{H}\right.$ thioph.), 8.17 (d, $1 \mathrm{H}, \mathrm{J}^{\text {ortho }}=8 \mathrm{~Hz}, 9-\mathrm{H}$ thioph.), $8.26\left(\mathrm{~d}, 1 \mathrm{H}, \mathrm{J}^{\text {meta }}=1 \mathrm{~Hz}, 6-\mathrm{H}\right.$ thioph.); ${ }^{13} \mathrm{C}$ NMR (DMSO-d6, $125.75 \mathrm{MHz}$ ), $\delta: 13.8$, 
$19.8,30.4,42.8,106.5,113.1,115.7,118.9,124.2,126.30,126.7,127.2,128.1,128.7,129.6$, $130.6,130.8,132.1,136.6,137.0,137.3,139.3,140.4,146.0 ; \mathrm{IR}\left(\mathrm{KBr}, v \mathrm{~cm}^{-1}\right): 3390,3040$, 2970, 2940, 2875, 1600, 1575, 1500, 1455, 1415, 1300, 1220, 1160, 1065, 970, 915, 880, 835, 760, 720, 700, 640; FLU (toluene, $\lambda_{\max }, \mathrm{nm}, \lambda_{\mathrm{EX}}=387.0 \mathrm{~nm}$ ) 473.0.

1-Dimethylamino-3, 7-bis [(E)-2-phenylethenyl]-5H-dibenzo $[b, d]$ thiophene-5,5-dione (19). According to the procedure for obtaining of 17: triphenylbenzylphosphonium bromide (0.9 g, 2 $\mathrm{mmol})$, t-BuOK $(0.25 \mathrm{~g}, 2.2 \mathrm{mmol})$, the crude product $16(0.3 \mathrm{~g})$. After purification 19 (246 $\mathrm{mg}$, $11 \%$ calculated from diester 11) was obtained as a yellow solid. $\mathrm{MP}=240-242^{\circ} \mathrm{C}$ (toluene). Elemental Anal. Calcd for $\mathrm{C}_{30} \mathrm{H}_{25} \mathrm{NO}_{2} \mathrm{~S}$ : C 77.72, H 5.44, N 3.02, S 6.92. Found: C 77,96, H 5.32, N 2.88, S 7.15; ${ }^{1} \mathrm{H}$ NMR (DMSO-d6, $\left.500 \mathrm{MHz}\right), \delta: 3.26\left(\mathrm{~s}, 6 \mathrm{H}, \mathrm{N}\left(\mathrm{CH}_{3}\right)_{2}\right), 7.72-7.85$ (m, $8 \mathrm{H}$, arom.), $8.01(\mathrm{~d}, 2 \mathrm{H}, \mathrm{J}=16 \mathrm{~Hz}$, trans $-\mathbf{C H}=\mathrm{CH}), 8.01-8.10(\mathrm{~m}, 5 \mathrm{H}$, arom.), $8.36(\mathrm{~s}, 1 \mathrm{H}, 4-\mathrm{H}$ dibenzothioph.), $8.38\left(\mathrm{dd}, 1 \mathrm{H}, \mathrm{J}^{\text {ortho }}=8 \mathrm{~Hz}, \mathrm{~J}^{\text {meta }}=1.5 \mathrm{~Hz}, 8-\mathrm{H}\right.$ dibenzothioph.), $8.66(\mathrm{~d}, 1 \mathrm{H}$, $\mathrm{J}^{\text {ortho }}=8 \mathrm{~Hz}, 9-\mathrm{H}$ dibenzothioph. $), 8,70\left(\mathrm{~d}, 1 \mathrm{H}, \mathrm{J}^{\text {meta }}=1.5 \mathrm{~Hz}, 6-\mathrm{H}\right.$ dibenzothioph. $) ;{ }^{13} \mathrm{C}$ NMR (DMSO-d6, $125.75 \mathrm{MHz}$ ), $\delta: 21.0,30.6,43.9,112.7,118.8,121.0,122.9,125.2,125.6,126.3$, $126.5,126.8,128.1,128.2,128.9,131.1,131.4,132.4,136.5,136.6,137.5,138.5,139.7,140.6$, 151.7; IR $\left(\mathrm{KBr}, v \mathrm{~cm}^{-1}\right): 3075,3090,2960,2880,2855,2810,1630,1600,1485,1460,1410$, $1305,1215,1165,1070,1015,975,945,900,845,765,730,705,640$; FLU (toluene, $\lambda_{\max }, \mathrm{nm}$, $\left.\lambda_{\mathrm{EX}}=406.0 \mathrm{~nm}\right) 472.0$.

Dibutyl 2, 2'-dihydroxy [1, 1'-biphenyl]-4, 4'-dicarboxylate (21a). A mixture of well ground $\mathrm{NaOH}(10 \mathrm{~g}, 0.25 \mathrm{~mol})$ and the diacid $8(1 \mathrm{~g}, 3 \mathrm{mmol})$ was heated at $210^{\circ} \mathrm{C}$ under intensive stirring for $20 \mathrm{~min}$. Then the cooled fuse was dissolved in $100 \mathrm{ml}$ of water and was acidified with $10 \%$ hydrochloric acid. Precipitated crystals were filtered off, washed with water and air-dried. The crude product was boiled in mixture of $30 \mathrm{ml}$ of butanol and $0.5 \mathrm{ml}$ of $\mathrm{H}_{2} \mathrm{SO}_{4}$ with regular removing of nascent water until TLC indicated no starting material. The reaction mixture was diluted with $\mathrm{CHCl}_{3}$, was washed with $100 \mathrm{ml}$ of $5 \% \mathrm{NaHCO}_{3}$ and brine. Concentration of the solution in vacuum afforded a brown solid, which was placed in the Soxhlet apparatus and was extracted with petroleum ether to remove 2-hydroxy derivative $\mathbf{2 1 b}$. The solid residue was recrystallazed from toluene to yield 21 a $(0.6 \mathrm{~g}, 52 \%)$ as white crystals. The extract was concentrated in vacuum and the residue was purified by column chromatography on silica gel (toluene) to give $\mathbf{2 1 b}(0.3 \mathrm{~g}, 27 \%)$ as a white solid.

Dibutyl 2, 2'-dihydroxy [1, 1'-biphenyl]-4, 4'-dicarboxylate (21a). $\mathrm{MP}=170-172{ }^{\circ} \mathrm{C}$; Elemental Anal. Calcd for $\mathrm{C}_{22} \mathrm{H}_{26} \mathrm{O}_{6}$ : C 68.38, H 6.78 Found: C 68.00, H 6.65; ${ }^{1} \mathrm{H}$ NMR (DMSO-d 6 , 500 $\mathrm{MHz}) \delta: 0.94\left(\mathrm{t}, 6 \mathrm{H}, \mathrm{J}=7.4 \mathrm{~Hz}, \mathrm{CH}_{3}\right), 1.40-1.47\left(\mathrm{~m}, 4 \mathrm{H}, \mathrm{CH}_{2}\right), 1.66-1.72\left(\mathrm{~m}, 4 \mathrm{H}, \mathrm{CH}_{2}\right), 4.27(\mathrm{t}$, $\left.4 \mathrm{H}, \mathrm{J}=6.5 \mathrm{~Hz},-\mathrm{OCH}_{2}\right), 7.29\left(\mathrm{~d}, 2 \mathrm{H}, \mathrm{J}^{\text {ortho }}=8 \mathrm{~Hz}, 6-, 6^{\prime}-\mathrm{H}\right), 7.42\left(\mathrm{dd}, 2 \mathrm{H}, \mathrm{J}^{\text {ortho }}=8 \mathrm{~Hz}, \mathrm{~J}^{\text {meta }}=1\right.$ Hz, 5-, 5'-H), 7.52 (br s, 2H, 3-, 3'-H), 9.80 (s, 2H, OH); IR (KBr, v cm ${ }^{-1}$ ): 3450, 2980, 2960, 2900, 1730, 1630, 1600, 1490, 1450, 1440, 1420, 1320, 1260, 1240, 1150, 1130, 1090, 1050, 1040, 990, 920, 870, 840, 790, 640.

Dibutyl 2-hydroxy [1,1'-biphenyl]-4,4'-dicarboxylate (21b). $\mathrm{MP}=93-95^{\circ} \mathrm{C}$; Elemental Anal. Calcd for $\mathrm{C}_{22} \mathrm{H}_{26} \mathrm{O}_{5}$ : C 71.33, H 7.07 Found: C 70.97, H 6.99; ${ }^{1} \mathrm{H}$ NMR (DMSO-d 6 , $\left.500 \mathrm{MHz}\right) \delta$ : $0.94\left(\mathrm{t}, 6 \mathrm{H}, \mathrm{J}=7.4 \mathrm{~Hz}, \mathrm{CH}_{3}\right), 1.40-1.47\left(\mathrm{~m}, 4 \mathrm{H}, \mathrm{CH}_{2}\right), 1.66-1.73\left(\mathrm{~m}, 4 \mathrm{H}, \mathrm{CH}_{2}\right), 4.27(\mathrm{t}, 4 \mathrm{H}, \mathrm{J}=$ 
$\left.6.5 \mathrm{~Hz},-\mathrm{OCH}_{2}\right), 4.29\left(\mathrm{t}, 4 \mathrm{H}, \mathrm{J}=6.5 \mathrm{~Hz},-\mathrm{OCH}_{2}\right), 7.45\left(\mathrm{~d}, 1 \mathrm{H}, \mathrm{J}^{\text {ortho }}=8 \mathrm{~Hz}, 5-\right.$ or 6-H), 7.49 (d, $1 \mathrm{H}, \mathrm{J}^{\text {ortho }}=8 \mathrm{~Hz}, 5-$ or $\left.6-\mathrm{H}\right), 7.60($ br s, $1 \mathrm{H}, 3-\mathrm{H}), 7.74\left(\mathrm{~d}, 2 \mathrm{H}, \mathrm{J}^{\text {ortho }}=8.2 \mathrm{~Hz}, 3^{\prime}-, 5^{\prime}-\mathrm{H}\right), 8.00(\mathrm{~d}$, $\left.2 \mathrm{H}, \mathrm{J}^{\text {ortho }}=8.2 \mathrm{~Hz}, 2^{\prime}-, 6^{\prime}-\mathrm{H}\right), 10.22(\mathrm{~s}, 1 \mathrm{H}, \mathrm{OH})$; IR $\left(\mathrm{KBr}, v_{\mathrm{cm}}{ }^{-1}\right): 3430,2990,2960,2910$, $1740,1710,1630,1610,1500,1450,1430,1380,1320,1290,1260,1170,1150,1140,1100$, $1070,1070,1040,1020,970,930,900,850,820,800,770,740,650$.

2-Hexyloxy-4, 4'-bis [hydroxymethyl]-1,1'-biphenyl (23). To dry methanol (11) saturated with $\mathrm{HCl}, 26.5 \mathrm{~g}(0.103 \mathrm{~mol})$ of well-milled powder of 2-hydroxy [1,1'-biphenyl]-4,4'-dicarboxylic acid 20 was added. The resulting suspension was stirred under reflux for $12 \mathrm{~h}$. After cooling, a precipitate was filtered off, washed with cold methanol. The crude product was dissolved in 180 $\mathrm{ml}$ of DMSO and $13.8 \mathrm{~g}(0.1 \mathrm{~mol})$ of $\mathrm{K}_{2} \mathrm{CO}_{3}$ was added. The reaction mixture was heated at $75^{\circ} \mathrm{C}, 16.5 \mathrm{~g}(0.1 \mathrm{~mol})$ of 1-bromohexane was added and the resulting solution was stirred for 10 $\mathrm{h}$ at $75^{\circ} \mathrm{C}$, then was poured into 0.51 of $5 \%$ hydrochloric acid, and extracted with ethyl acetate. The organic layer was washed with water and dried over $\mathrm{Na}_{2} \mathrm{SO}_{4}$. After removal of solvent, the residue was recrystallized from ethanol. The obtained crystals were dissolved in $150 \mathrm{ml}$ of THF and added dropwise to the suspension of $3.8 \mathrm{~g}(0.1 \mathrm{~mol}) \mathrm{LiAlH}_{4}$ in $100 \mathrm{ml}$ of THF under stirring. The reaction mixture was refluxed for $2 \mathrm{~h}$, excess of $\mathrm{LiAlH}_{4}$ was quenched with addition of 15 $\mathrm{ml}$ of $15 \%$ water solution of $\mathrm{NaOH}$, and an inorganic solid was separated by filtration and washed with THF. Solvent was removed under reduced pressure, the residue was dissolved in ethyl acetate, washed with water and dried over $\mathrm{Na}_{2} \mathrm{SO}_{4}$. After removal of solvent, the residue was crystallized from toluene to afford $26.73 \mathrm{~g}(83 \%)$ of white crystals. $\mathrm{MP}=60-61{ }^{\circ} \mathrm{C}$; Elemental Anal. Calcd for $\mathrm{C}_{20} \mathrm{H}_{26} \mathrm{O}_{3}$ : C 76.40, $\mathrm{H} 8.33$ Found: $\mathrm{C} 76.04, \mathrm{H} \mathrm{8.21} ;{ }^{1} \mathrm{H} \mathrm{NMR}\left(\mathrm{CDCl}_{3}\right.$, $\left.500 \mathrm{MHz}), \delta: 0.85\left(\mathrm{t}, \mathrm{J}=7 \mathrm{~Hz}, 3 \mathrm{H},-\mathrm{CH}_{3}\right), 1.4-1.48\left(\mathrm{~m}, 6 \mathrm{H},-\left(\mathrm{CH}_{2}\right)_{3}\right)^{-}\right), 1.58-1.86\left(\mathrm{~m}, 2 \mathrm{H},-\mathrm{CH}_{2^{-}}\right.$ ), 1.81 (s. $2 \mathrm{H},-\mathrm{OH}$ ), 3.95 (t, $\left.2 \mathrm{H}, \mathrm{J}=7 \mathrm{~Hz},-\mathrm{O}-\mathrm{CH}_{2^{-}}\right), 4.69$ (s, 2H, $\left.-\mathrm{CH}_{2^{-}}\right), 4.70$ (s, 2H, $-\mathrm{CH}_{2^{-}}$), $7.00\left(\mathrm{~d}, 1 \mathrm{H}, \mathrm{J}^{\text {meta }}=1.5 \mathrm{~Hz}, 3\right.$ biphenyl), $7.10\left(\mathrm{dd}, 1 \mathrm{H}, \mathrm{J}^{\text {ortho }}=7.5 \mathrm{~Hz}, \mathrm{~J}^{\text {meta }}=1.5 \mathrm{~Hz}, 5\right.$ biphenyl), 7.29 (d, 1H, J=7.5 Hz, 6 biphenyl), 7.41 (d, 2H, J ${ }^{\text {ortho }}=8.5 \mathrm{~Hz}, 3^{\prime}, 5$ ' biphenyl), 7.50 (d, $1 \mathrm{H}, \mathrm{J}^{\text {ortho }}$ $=8.5 \mathrm{~Hz}, 2^{\prime}, 6$ ' biphenyl); IR (KBr, $\left.v \mathrm{~cm}^{-1}\right)$ : 3350, 2950, 2880, 1610, 1580, 1490, 1470, 1430, 1400, 1270, 1175, 1140, 1010, 960, 820, 810; EIMS (m/z): 315([M+1] $\left.]^{+}, 10\right), 314\left([\mathrm{M}]^{+}, 44\right)$, $312\left([\mathrm{M}-2 \mathrm{H}]^{+}, 100\right), 311\left([\mathrm{M}-3 \mathrm{H}]^{+}, 71\right), 228\left(\left[\mathrm{C}_{14} \mathrm{H}_{12} \mathrm{O}_{3}\right]^{+}, 93\right), 226(61), 225(86), 209(50), 197(52)$, 153(43), 57(43), 43(42), 18(14).

2, 2'-Dihexyloxy-4, 4'-bis [hydroxymethyl]-1, 1'-biphenyl (24). To a solution of dibutyl 2, 2'dihydroxy [1, 1'-biphenyl]-4, 4'-dicarboxylate $21 \mathrm{a}(0.6 \mathrm{~g}, 1.5 \mathrm{mmol})$ and 1-bromohexane $(0.6 \mathrm{~g}$, $3.6 \mathrm{mmol})$ in $40 \mathrm{ml}$ of DMSO, anhydrous $\mathrm{K}_{2} \mathrm{CO}_{3}(0.5 \mathrm{~g}, 3.6 \mathrm{mmol})$ was added and the resulting mixture was heated at $60^{\circ} \mathrm{C}$ and stirred for $12 \mathrm{~h}$. Then the reaction mixture was diluted with water and extracted with chloroform. The combined organic solution was dried over $\mathrm{Na}_{2} \mathrm{SO}_{4}$, and then concentrated in vacuum. The residue brown oil was added as a solution in $10 \mathrm{ml}$ of dry THF to a suspension of $\mathrm{LiAlH}_{4}(0.37 \mathrm{~g}, 10 \mathrm{mmol})$ in $30 \mathrm{ml}$ of THF and the reaction mixture was stirred at RT for $12 \mathrm{~h}$. Excess of $\mathrm{LiAlH}_{4}$ was quenched with $5 \%$ solution of $\mathrm{NaOH}$ in water and inorganic solids were filtered off and washed with hot THF. After concentration of the filtrate in vacuum the residue was purified by column chromatography on silica gel (toluene) to yield 24 $(0.7 \mathrm{~g}, 80 \%)$ as colorless oil. Elemental Anal. Calcd for $\mathrm{C}_{26} \mathrm{H}_{38} \mathrm{O}_{4}$ : C 75.32, H 9.24 Found: C 
74.97, H 9.12; ${ }^{1} \mathrm{H}$ NMR (DMSO-d 6 , $\left.500 \mathrm{MHz}\right), \delta: 0.86\left(\mathrm{t}, 6 \mathrm{H}, \mathrm{J}=7 \mathrm{~Hz}, \mathrm{CH}_{3}\right), 1.20-1.32(\mathrm{~m}$, $\left.12 \mathrm{H}, \mathrm{CH}_{2}\right), 1.55-1.61\left(\mathrm{~m}, 4 \mathrm{H}, \mathrm{CH}_{2}\right), 3.88\left(\mathrm{t}, 4 \mathrm{H}, \mathrm{J}=6.5 \mathrm{~Hz},-\mathrm{OCH}_{2}-\right), 4.70(\mathrm{~d}, 4 \mathrm{H}, \mathrm{J}=5.5$, $\left.\mathrm{CH}_{2} \mathrm{OH}\right), 4.93(\mathrm{t}, 2 \mathrm{H}, \mathrm{J}=5.5 \mathrm{~Hz}, \mathrm{OH}), 6.82\left(\mathrm{dd}, 2 \mathrm{H}, \mathrm{J}^{\text {ortho }}=8 \mathrm{~Hz}, \mathrm{~J}^{\text {meta }}=1 \mathrm{~Hz}, 5-, 5\right.$ '-H), 6.90 (br s, 2H, 3-, 3'-H), 7.04 (d, 2H, J ortho $\left.^{\prime}=8 \mathrm{~Hz}, 6-, 6^{\prime}-\mathrm{H}\right)$; IR $\left(\mathrm{KBr}, \mathrm{v} \mathrm{cm}^{-1}\right)$ : 2975, 2950, 2880, $1620,1590,1570,1510,1470,1420,1290,1260,1200,1150,1070,1040,1025,970,940,865$, 840,790 .

4,4'-Bis[bromomethyl]-2-(hexyloxy)biphenyl. To a cooled at $0^{\circ} \mathrm{C}$ solution of $4 \mathrm{~g}(13 \mathrm{mmol})$ 4,4 -bis(dihydroxymethyl)-2-(hexyloxy)biphenyl (24) in $\mathrm{CCl}_{4}, 3 \mathrm{~g}$ (10 mmol) of $\mathrm{PBr}_{3}$ was added dropwise under stirring. The reaction mixture was stirred for $5 \mathrm{~h}$ at $\mathrm{RT}$, and then it was poured into $100 \mathrm{ml}$ of water, and extracted with $\mathrm{CCl}_{4}$. The combined organic solution was washed with water and dried over $\mathrm{Na}_{2} \mathrm{SO}_{4}$. After removal of solvent, the residue was purified by column chromatography on silica gel (eluent: toluene) to give white solid (4.89 g, 86\%), which was recrystallized from hexane. $\mathrm{MP}=40^{\circ} \mathrm{C}$; Elemental Anal. Calcd for $\mathrm{C}_{20} \mathrm{H}_{24} \mathrm{Br}_{2} \mathrm{O}$ : C 54.57, $\mathrm{H} 5.50$ Found: C 54.21, H 5.36; ${ }^{1} \mathrm{H}$ NMR $\left(\mathrm{CDCl}_{3}, 500 \mathrm{MHz}\right), \delta: 0.90\left(\mathrm{t}, \mathrm{J}=7 \mathrm{~Hz}, 3 \mathrm{H},-\mathrm{CH}_{3}\right), 1.2-1.9$ $\left(\mathrm{m}, 8 \mathrm{H},-\left(\mathrm{CH}_{2}\right)_{4}-\right), 3.96\left(\mathrm{t}, 2 \mathrm{H}, \mathrm{J}=7 \mathrm{~Hz},-\mathrm{OCH}_{2}-\right), 4.50\left(\mathrm{~s}, 2 \mathrm{H},-\mathrm{CH}_{2}-\right), 4.53\left(\mathrm{~s}, 2 \mathrm{H}, \mathrm{CH}_{2}-\mathrm{Br}\right), 6.99$ $\left(\mathrm{d}, 1 \mathrm{H}, \mathrm{J}^{\text {meta }}=1.5 \mathrm{~Hz}, 3-\mathrm{H}\right), 7.02\left(\mathrm{dd}, 1 \mathrm{H}, \mathrm{J}^{\text {ortho }}=7.5 \mathrm{~Hz}, \mathrm{~J}^{\text {meta }}=1.5 \mathrm{~Hz}, 5-\mathrm{H}\right), 7.27\left(\mathrm{~d}, 1 \mathrm{H}, \mathrm{J}^{\text {ortho }}=\right.$ $7.5 \mathrm{~Hz}, 6-\mathrm{H}), 7.39$ (d, 2H, J $\left.{ }^{\text {ortho }}=8.5 \mathrm{~Hz}, 3^{\prime}-, 5^{\prime}-\mathrm{H}\right), 7.48$ (d, 1H, J ${ }^{\text {ortho }}=8.5 \mathrm{~Hz}, 2^{\prime}$ '-, 6'-H); IR $\left(\mathrm{KBr}, v \mathrm{~cm}^{-1}\right): 3450,2930,2860,1610,1500,1440,1400,1280,1210,1180,1070,820,650$, 600,550 .

4, 4'-bis [(triphenylphosphonio)methyl]-2-(hexyloxy)biphenyl dibromide (25). A mixture of $2 \mathrm{~g}$ (4,5 mmol) 4,4'-bis(bromomethyl)-2-(hexyloxy)biphenyl, $3.33 \mathrm{~g}$ (12 mmol) of triphenylphosphine, and $50 \mathrm{ml}$ of DMF was heated at $150^{\circ} \mathrm{C}$ for $5-6 \mathrm{~h}$. Then a half volume of DMF was removed in vacuum and $70 \mathrm{ml}$ of toluene was added. The precipitated white crystals were filtered off, washed with cold toluene $(2 \times 10 \mathrm{ml})$, and vacuum dried at room temperature to afford $4.18 \mathrm{~g}(95 \%)$ of a white powder. $\mathrm{MP}=250^{\circ} \mathrm{C}\left(\mathrm{dec}\right.$ ); $\mathrm{IR}\left(\mathrm{KBr}, \mathrm{cm}^{-1}\right): 3450,2950,2850$, 1440, 1120, 750, 720, 680, 500.

2-Hexyloxy-1, 1'-biphenyl-4,4'-dicarbaldehyde (26). To a stirred solution of 2-hexyloxy-4,4'bis[hydroxymethyl]-1,1'-biphenyl 23 (14 g, $0.041 \mathrm{~mol})$ in $\mathrm{CH}_{2} \mathrm{Cl}_{2}, 26.4 \mathrm{~g}(0.123 \mathrm{~mol})$ of PCC in $80 \mathrm{ml}$ of dioxane was added dropwise during $1.5 \mathrm{~h}$. Inorganic solids were separated off, the filtrate was concentrated in vacuum and the residue was purified by column chromatography on silica gel to give 2-(hexyloxy)-1,1'-biphenyl-4,4'-dicarbaldehyde 26 (12.02 g, 85\%) as a white solid. Recrystallization from hexane gave yellow crystals. $\mathrm{MP}=40-41^{\circ} \mathrm{C}$; Elemental Anal. Calcd for $\mathrm{C}_{20} \mathrm{H}_{22} \mathrm{O}_{3}$ : C 77.39, $\mathrm{H} 7.14$ Found: C 76.99, $\mathrm{H} 7.04 ;{ }^{1} \mathrm{H} \mathrm{NMR}\left(\mathrm{CDCl}_{3}, 500 \mathrm{MHz}\right), \delta$ : 0.85 (t, $\left.\mathrm{J}=7 \mathrm{~Hz}, 3 \mathrm{H},-\mathrm{CH}_{3}\right), 1.1-1.8\left(\mathrm{~m}, 8 \mathrm{H},-\left(\mathrm{CH}_{2}\right)_{4}-\right), 4.05\left(\mathrm{t}, 2 \mathrm{H}, \mathrm{J}=7 \mathrm{~Hz},-\mathrm{O}-\mathrm{CH}_{2}-\right), 7.38\left(\mathrm{~d}, 1 \mathrm{H}, \mathrm{J}^{\text {meta }}\right.$ $=1.5 \mathrm{~Hz}, 3$ biphenyl), $7.43\left(\mathrm{dd}, 1 \mathrm{H}, \mathrm{J}^{\text {ortho }}=7.5 \mathrm{~Hz}, \mathrm{~J}^{\text {meta }}=1.5 \mathrm{~Hz}, 5\right.$ biphenyl), $7.56(\mathrm{~d}, 1 \mathrm{H}, \mathrm{J}=$ $7.5 \mathrm{~Hz}, 6$ biphenyl), $7.83\left(\mathrm{~d}, 2 \mathrm{H}, \mathrm{J}^{\text {ortho }}=8.5 \mathrm{~Hz}, 3^{\prime}, 5^{\prime}\right.$ biphenyl), 7.87 (d, 1H, J ${ }^{\text {ortho }}=8.5 \mathrm{~Hz}, 2^{\prime}, 6^{\prime}$ biphenyl), 10.05 (s, 2H, CHO); IR (KBr, $v \mathrm{~cm}^{-1}$ ): 2960, 2940, 2860, 1700, 1600, 1570, 1560, 1430, 1380, 1310, 11290, 1270, 1210, 1170, 1150, 1140, 1050, 1000, 840, 810, 760; EIMS (m/z) : 311([M+1] $\left.]^{+}, 16\right), 310\left([\mathrm{M}]^{+}, 100\right)$. 
2, 2'-Bis (hexyloxy) [1,1'-biphenyl]-4,4'-dicarbaldehyde (27). To a solution of 24 (2.7 g, 6.5 $\mathrm{mmol}$ ) in $70 \mathrm{ml}$ of $\mathrm{CHCl}_{3}, 5 \mathrm{~g} \mathrm{PCC}$ in $50 \mathrm{ml}$ of dioxane was added and the resulting mixture was stirred at RT for $10 \mathrm{~h}$. The reaction mixture was filtered through a layer of silica gel and purified by flash chromatography to afford 27 as colorless oil. Yield $2.2 \mathrm{~g} \mathrm{(82 \% ).} \mathrm{Elemental} \mathrm{Anal.} \mathrm{Calcd}$ for $\mathrm{C}_{26} \mathrm{H}_{34} \mathrm{O}_{4}$ : C 76.06, $\mathrm{H} 8.35$ Found: $\mathrm{C} 75.74, \mathrm{H} 8.23 ;{ }^{1} \mathrm{H} \mathrm{NMR}\left(\mathrm{CDCl}_{3}, 500 \mathrm{MHz}\right), \delta: 0.82(\mathrm{t}$, $6 \mathrm{H}, \mathrm{J}=7 \mathrm{~Hz}), 1.22-1.30\left(\mathrm{~m}, 12 \mathrm{H},-\mathrm{CH}_{2}-\right), 1.58-163\left(\mathrm{~m}, 4 \mathrm{H},-\mathrm{CH}_{2^{-}}\right), 4.00(\mathrm{t}, 4 \mathrm{H}, \mathrm{J}=6.5 \mathrm{~Hz}$, $\left.\mathrm{OCH}_{2^{-}}\right), 7.37$ (d, 2H, J = 7.5 Hz, 6,6'-H), 7.45 (d, 2H, J = 1 Hz, 3,3'-H), 7.51 (dd, 2H, J = 1, Hz, $\left.\mathrm{J}=7.5 \mathrm{~Hz}, 5,5^{\prime}-\mathrm{H}\right), 9.98$ (s, 2H, CHO); IR (KBr, $\left.v \mathrm{~cm}^{-1}\right): 3460,3000,2970,2895,1730,1690$, $1610,1570,1485,1435,1400,1280,1260,1230,1185,1155,1055,1025,920,890,850,760$, 680.

4, 4'-Bis [(E)-2-(1,3-benzoxazol-2-yl)ethenyl]-2-(hexyloxy)biphenyl (28). A sodium methylate solution, obtained from $0.32 \mathrm{~g}$ of sodium $(14 \mathrm{mmol})$ and $15 \mathrm{ml}$ of $\mathrm{MeOH}$ was added dropwise under stirring to a solution of aldehyde 26 (1.86 g, $6 \mathrm{mmol})$ and 2-methyl-1,3-benzoxazole (2.1 $\mathrm{g}, 15 \mathrm{mmol}$ ) in $25 \mathrm{ml}$ of DMSO. The reaction mixture was heated at $50-60^{\circ} \mathrm{C}$ and stirred for $3 \mathrm{~h}$, then cooled at RT and a formed precipitate was filtered off, washed with $\mathrm{MeOH}$ and dried in vacuum. Recrystallization from ethanol gave $28(2.6 \mathrm{~g}, 80 \%)$ as yellow crystals. $\mathrm{MP}=155-$ $156^{\circ} \mathrm{C}$; Elemental Anal. Calcd for $\mathrm{C}_{36} \mathrm{H}_{32} \mathrm{~N}_{2} \mathrm{O}_{3}$ : C 79.97, H 5.97, N 5.18 Found: C 80.32, H 5.73, $\mathrm{N} 5.33 ;{ }^{1} \mathrm{H}$ NMR $\left(\mathrm{CDCl}_{3}, 500 \mathrm{MHz}\right) \delta: 0.900\left(\mathrm{t}, 3 \mathrm{H}, \mathrm{J}=6.4 \mathrm{~Hz},-\mathrm{CH}_{3}\right), 1.30-1.50(\mathrm{~m}, 6 \mathrm{H}$, $\left.\left(\mathrm{CH}_{2}\right)_{3^{-}}\right), 1.80\left(\mathrm{~m}, 2 \mathrm{H},-\mathrm{CH}_{2^{-}}\right) 4.11\left(\mathrm{t}, 2 \mathrm{H}, \mathrm{J}=6.4 \mathrm{~Hz},-\mathrm{OCH}_{2^{-}}\right), 7.11(\mathrm{~d}, 1 \mathrm{H} \mathrm{J}=16 \mathrm{~Hz}$, trans$\mathrm{CH}=\mathrm{CH}), 7.12(\mathrm{~d}, 1 \mathrm{H} \mathrm{J}=16 \mathrm{~Hz}$, trans $-\mathrm{CH}=\mathrm{CH}), 7.19(\mathrm{~s}, 1 \mathrm{H}$, arom.), $7.27(\mathrm{~d}, 1 \mathrm{H}, \mathrm{J}=7.2 \mathrm{~Hz}$, arom.), 7.33- 7.36 (m, 4H, arom.), 7.41 (d, 1H, J= 8 Гц, arom.), 7.53- 7.55 (m, 2H, arom.), 7.637.67 (m, 4H, arom.), 7.72-7.74 (m, 2H, arom.), 7.79 (d, 1H, J = 16.0 Hz, trans-CH=CH), 7.83 (d, $1 \mathrm{H}, \mathrm{J}=16.0 \mathrm{~Hz}$, trans-CH=CH); IR $\left(\mathrm{KBr}, v \mathrm{~cm}^{-1}\right): 2950,2870,1640,1600,1540,1460,1430$, $1410,1360,1270,1250,1190,1160,1020,980,940,850,830,760 ; \mathrm{FLU}\left(\mathrm{CHCl}_{3}, \lambda_{\max }, \mathrm{nm}, \lambda_{\mathrm{EX}}\right.$ $=365.0 \mathrm{~nm}) 449.0$, film -475 .

4, 4'-Bis [(E)-2-(1,3-benzoxazol-2-yl)ethenyl]-2,2'-(dihexyloxy)biphenyl (29). To a solution of dialdehyde (27) (200 mg, $0.5 \mathrm{mmol}$ ) and 2-methyl-1, 3-benzoxazole (230 mg, $1.7 \mathrm{mmol})$ in 15.0 $\mathrm{ml}$ of DMSO, t-BuOK (120 mg, $1 \mathrm{mmol})$ was added. The reaction mixture was heated at $40-$ $50^{\circ} \mathrm{C}$ for $16 \mathrm{~h}$ under stirring, then was diluted with brine and extracted with toluene. Organic phases were combined and solvents removed by the rotary evaporator. The residue was purified by column chromatography on silica gel (toluene) to give 29 (260 mg, 80\%) as yellow solid. The recrystallization from toluene afforded yellow crystals. $\mathrm{MP}=128-130^{\circ} \mathrm{C}$; Elemental Anal. Calcd for $\mathrm{C}_{42} \mathrm{H}_{44} \mathrm{~N}_{2} \mathrm{O}_{4}$ : C 78.72, H 6.92, N 4.37 Found: C 78.43, H 6.99, N 4.15; ${ }^{1} \mathrm{H}$ NMR (DMSO-d 6 , $500 \mathrm{MHz}), \delta: 0.85\left(\mathrm{t}, 6 \mathrm{H}, \mathrm{J}=7 \mathrm{~Hz}, \mathrm{CH}_{3}\right), 1.21-1.35\left(\mathrm{~m}, 12 \mathrm{H},-\mathrm{CH}_{2^{-}}\right), 1.63\left(\mathrm{~m}, 4 \mathrm{H},-\left(\mathrm{CH}_{2}\right)_{2^{-}}\right)$, $4.01\left(\mathrm{t}, 4 \mathrm{H}, \mathrm{J}=6.5 \mathrm{~Hz},-\mathrm{OCH}_{2}\right), 7.22-7.27$ (m, 6H, arom.), 7.31-7.36 (m, 6H, arom.), 7.59 (dd, $\left.2 \mathrm{H}, \mathrm{J}^{\text {ortho }}=7 \mathrm{~Hz}, \mathrm{~J}^{\text {meta }}=1.5 \mathrm{~Hz}, 6,6^{\prime}-\mathrm{H}\right), 7.67\left(\mathrm{dd}, 2 \mathrm{H}, \mathrm{J}^{\text {ortho }}=7 \mathrm{~Hz}, \mathrm{~J}^{\text {meta }}=2 \mathrm{~Hz}, 5,5^{\prime}-\mathrm{H}\right), 7.79(\mathrm{~d}$, $2 \mathrm{H}, \mathrm{J}=16 \mathrm{~Hz}$, trans-CH=CH- $) ;{ }^{13} \mathrm{C} \mathrm{NMR}\left(\mathrm{CDCl}_{3}, 125.75 \mathrm{MHz}\right), \delta: 13.96,14.11,22.58,22.68$, $25.64,29.09,31.45,31.87,68.49,110.30,110.49,113.67,119.82,124.51,125.19,129.38$, 131.94, 135.61, 139.58, 142.21, 150.43, 156.89; IR (KBr, $\left.v \mathrm{~cm}^{-1}\right): 3070,2970,2870,1645,1605$, 
$1540,1495,1455,1420,1395,1355,1250,1275,1250,1190,1160,1040,1020,985,950,870$, 830, 810, 765; FLU $\left(\mathrm{CHCl}_{3}, \lambda_{\max }, \mathrm{nm}, \lambda_{\mathrm{EX}}=365.0 \mathrm{~nm}\right) 455.0$; film - 443.

\section{General procedure for the Wittig condensation}

To the suspension of $1 \mathrm{~g}$ (1 mmol) 4,4'-bis[(triphenylphosphonio)methyl]-2-(hexyloxy)biphenyl dibromide (25) in $20 \mathrm{ml}$ of dry THF, $t$-BuOK $(0.28 \mathrm{~g}, 2.5 \mathrm{mmol})$ was added and the reaction mixture was stirred until complete dissolution of phosphonium salt happened with formation of the dark-red solution. Then a solution of $2 \mathrm{mmol}$ corresponding aldehyde in $10 \mathrm{ml}$ of THF was slowly added and the resulting mixture was stirred for an additional $2 \mathrm{~h}$. After $2 \mathrm{~h}$, the reaction mixture was poured into $100 \mathrm{ml}$ of water and extracted with chloroform. The combined organic phase was washed with water and then dried over $\mathrm{Na}_{2} \mathrm{SO}_{4}$. After removal of solvent, the residue was purified by column chromatography on silica gel (eluent: chloroform) and recrystallized from hexane.

2-Hexyloxy-4, 4'-bis [2-phenylethenyl]-1,1'-biphenyl (30a). Yield 0.44 g (95\%). MP = 152$153^{\circ} \mathrm{C}$; Elemental Anal. Calcd for $\mathrm{C}_{34} \mathrm{H}_{34} \mathrm{O}$ : C 89.04, H 7.47 Found: C 88.69, H 7.61; ${ }^{1} \mathrm{H}$ NMR $\left(\mathrm{CDCl}_{3}, 500 \mathrm{MHz}\right) \delta: 0.85\left(\mathrm{t}, 3 \mathrm{H}, \mathrm{J}=7 \mathrm{~Hz},-\mathrm{CH}_{3}\right), 1.2-1.9\left(\mathrm{~m}, 8 \mathrm{H},-\left(\mathrm{CH}_{2}\right)_{4}-\right), 4.05(\mathrm{t}, 2 \mathrm{H}, \mathrm{J}=7$ $\left.\mathrm{Hz},-\mathrm{O}-\mathrm{CH}_{2}-\right), 7.1-7.6$ (m, 21H, arom.); ${ }^{13} \mathrm{C} \mathrm{NMR}\left(\mathrm{CDCl}_{3}, 125.75 \mathrm{MHz}\right), \delta: 14.01,22.58,25.78$, $29.14,31.45,68.50,110.30,119.30,126.03,126.31,126.47,127.52,127.66,128.38,128.47$, $128.52,128.67,128.69,129.76,137.24,137.44,137.85,137.65,137.80 ;\left(\mathrm{KBr}, v \mathrm{~cm}^{-1}\right): 2950$, 2930, 2850, 1600, 1500, 1450, 1400, 1270, 1230, 1180, 1140, 1070, 1030, 1000, 960, 810, 750, 680; UV $\left(\mathrm{CHCl}_{3}, \lambda_{\max }, \mathrm{nm}\left(\varepsilon, 1 /\left(\mathrm{mol}^{*} \mathrm{~cm}\right)\right)\right): 340.0$ (16300); FLU $\left(\mathrm{CHCl}_{3}, \lambda_{\max }, \mathrm{nm}, \lambda_{\mathrm{EX}}=365.0\right.$ nm) 428.0.

2-Hexyloxy-4, 4'-bis [2-(4-cyanophenyl)ethenyl]-1,1'-biphenyl (30b). Yield $0.3 \mathrm{~g}$ (61\%). MP $=170-171^{\circ} \mathrm{C}$; Elemental Anal. Calcd for $\mathrm{C}_{36} \mathrm{H}_{32} \mathrm{~N}_{2} \mathrm{O}$ : C 85.01, H 6.34, N 5.51 Found: C 84.48, $\mathrm{H} 7.52, \mathrm{~N} 5.66 ;{ }^{1} \mathrm{H} \mathrm{NMR}\left(\mathrm{CDCl}_{3}, 500 \mathrm{MHz}\right) \delta: 0.86\left(\mathrm{t}, 3 \mathrm{H}, \mathrm{J}=7 \mathrm{~Hz},-\mathrm{CH}_{3}\right), 1.3-1.8(\mathrm{~m}, 8 \mathrm{H}$, $\left.\left(\mathrm{CH}_{2}\right)_{4}-\right), 4.10\left(\mathrm{t}, 2 \mathrm{H}, \mathrm{J}=7 \mathrm{~Hz},-\mathrm{OCH}_{2}\right), 7.3-8.0(\mathrm{~m}, 19 \mathrm{H}$, aromatic $\mathrm{H}) ;{ }^{13} \mathrm{C} \mathrm{NMR}\left(\mathrm{CDCl}_{3}\right.$, $125.75 \mathrm{MHz}) \delta: 14.99,22.26,29.08,31.41,68.54,110.55,119.06,119.74,126.49,126.81$, $126.86,129.77,129.91,130.48,130.89,132.09,132.13,132.50,136.98,138.43,141.69,141.89$, 156.39; IR (KBr, $\left.v \mathrm{~cm}^{-1}\right): 2960,2940,2860,1610,1510,1450,1400,1270,1230,1180,1140$, 1070, 1030, 1000, 960, 810, 750, 680; UV $\left(\mathrm{CHCl}_{3}, \lambda_{\max }, \mathrm{nm}(\varepsilon, 1 /(\mathrm{mol} * \mathrm{~cm}))\right): 359.0(27000)$; FLU $\left(\mathrm{CHCl}_{3}, \lambda_{\text {max }}, \mathrm{nm}, \lambda_{\mathrm{EX}}=365.0 \mathrm{~nm}\right) 443.0$.

2-Hexyloxy-4, 4'-bis [2-(4-fluorophenyl)ethenyl]-1,1'-biphenyl (30c). Yield $0.31 \mathrm{~g}$ (63\%). MP $=104-105^{\circ} \mathrm{C}$; Elemental Anal. Calcd for $\mathrm{C}_{34} \mathrm{H}_{32} \mathrm{~F}_{2} \mathrm{O}$ : C 86.56, H 6.52 Found: $\mathrm{C} 86.87$, H 6.68; ${ }^{1} \mathrm{H}$ NMR $\left(\mathrm{CDCl}_{3}, 500 \mathrm{MHz}\right) \delta: 0.89\left(\mathrm{t}, 3 \mathrm{H}, \mathrm{J}=7 \mathrm{~Hz},-\mathrm{CH}_{3}\right), 1.3-1.8\left(\mathrm{~m}, 8 \mathrm{H},-\left(\mathrm{CH}_{2}\right)_{4}-\right), 4.03(\mathrm{t}$, $\left.2 \mathrm{H}, \mathrm{J}=7 \mathrm{~Hz},-\mathrm{O}-\mathrm{CH}_{2}-\right), 7.3-7.6(\mathrm{~m}, 19 \mathrm{H}$, arom. $) ;{ }^{13} \mathrm{C} \mathrm{NMR}\left(\mathrm{CDCl}_{3}, 125.75 \mathrm{MHz}\right) \delta$ : 14.0, 22.6, 25.8, 29.1, 31.4, 68.5, 110.2, 115.6, 119.2, 125.9, 127.2, 127.5, 127.9, 128.3, 129.8, 130.8, 135.5, 137.6, 156.3; IR (KBr, $\left.v \mathrm{~cm}^{-1}\right): 2960,2940,2860,1600,1520,1440,1400,1270,1240,1160$, 1140, 1010, 970, 850, 840, 760, 730, 700, 540; UV $\left(\mathrm{CHCl}_{3}, \lambda_{\max }, \mathrm{nm}\left(\varepsilon, 1 /\left(\mathrm{mol}^{*} \mathrm{~cm}\right)\right)\right): 395.0$ (23000); FLU ( $\left.\mathrm{CHCl}_{3}, \lambda_{\max }, \mathrm{nm}, \lambda_{\mathrm{EX}}=365.0 \mathrm{~nm}\right) 405.0$. 
2-Hexyloxy-4, 4'-bis \{(E)-2-[4-(diphenylamino)phenyl]ethenyl\}-1,1'-biphenyl (30d). Yield 0.6 g (75\%). $\mathrm{MP}=171-172^{\circ} \mathrm{C}$; Elemental Anal. Calcd for $\mathrm{C}_{58} \mathrm{H}_{52} \mathrm{~N}_{2} \mathrm{O}: \mathrm{C} 87.84, \mathrm{H} 6.61, \mathrm{~N} 3.53$ Found: C 87.58, $\mathrm{H}$ 6.79, N 3.62; ${ }^{1} \mathrm{H} \mathrm{NMR}\left(\mathrm{CDCl}_{3}, 500 \mathrm{MHz}\right), \delta: 0.89\left(\mathrm{t}, 3 \mathrm{H}, \mathrm{J}=7 \mathrm{~Hz},-\mathrm{CH}_{3}\right)$, 1.3- $1.8\left(\mathrm{~m}, 8 \mathrm{H},-\left(\mathrm{CH}_{2}\right)_{4}-\right), 4.03\left(\mathrm{t}, 2 \mathrm{H}, \mathrm{J}=7 \mathrm{~Hz},-\mathrm{OCH}_{2}-\right), 7.00(\mathrm{~d}, 2 \mathrm{H}, \mathrm{J}=16 \mathrm{~Hz}$, trans- $\underline{\mathrm{H}}=\mathrm{CH}-$ ), 7.0-7.2 (m, 18H), $7.15\left(\mathrm{dd}, 1 \mathrm{H}, \mathrm{J}^{\text {ortho }}=8 \mathrm{~Hz}, \mathrm{~J}^{\text {meta }}=1.5 \mathrm{~Hz}, 5-\mathrm{H}\right.$ biphenyl), $7.26\left(\mathrm{~d}, 2 \mathrm{H}, \mathrm{J}^{\text {ortho }}=\right.$ $16 \mathrm{~Hz}$, trans- $\mathrm{CH}=\mathrm{C} \underline{\mathrm{H}}-), 7.2-7.3(\mathrm{~m}, 7 \mathrm{H}), 7.34\left(\mathrm{~d}, 1 \mathrm{H}, \mathrm{J}^{\text {ortho }}=8 \mathrm{~Hz}, 6-\mathrm{H}\right.$ biphenyl), $7.40(\mathrm{~d}, 4 \mathrm{H}$, $\left.\mathrm{J}^{\text {ortho }}=9 \mathrm{~Hz}-\mathrm{C}_{6} \mathrm{H}_{4}-\mathrm{N}\right), 7.56\left(\mathrm{~d}, 2 \mathrm{H}, \mathrm{J}^{\text {ortho }}=9 \mathrm{~Hz}, 3^{\prime}-, 5^{\prime}-\mathrm{H}\right.$ biphenyl), $7.61\left(\mathrm{~d}, 2 \mathrm{H}, \mathrm{J}^{\text {ortho }}=9 \mathrm{~Hz}\right.$, 2'-, 6'-H biphenyl); ${ }^{13} \mathrm{C} \mathrm{NMR}\left(\mathrm{CDCl}_{3}, 125.75 \mathrm{MHz}\right), \delta: 14.2,22.7,25.9,29.3,31.6,68.6,110.3$, $119.2,123.1,123.2,123.7,123.8,124.6,124.7,126.0,126.5,126.9,127.0,127.48,127.53$, $128.0,128.4,129.4,129.9,130.9,131.5,131.8,136.2,137.5,138.3,147.4,147.5,147.6,147.7$, 156.5; IR (KBr, $\left.v \mathrm{~cm}^{-1}\right): 2940,2860,1580,1480,1320,1270,1180,970,830,750,700$; UV $\left(\mathrm{CHCl}_{3}, \lambda_{\max }, \mathrm{nm}(\varepsilon, 1 /(\mathrm{mol} * \mathrm{~cm}))\right): 382.0$ (45000); FLU $\left(\mathrm{CHCl}_{3}, \lambda_{\max }, \mathrm{nm}, \lambda_{\mathrm{EX}}=365.0 \mathrm{~nm}\right)$ 452.0.

2-[(2-ethylhexyl)oxy]-4,4'-bis\{(E)-2-[4-(diphenylamino)phenyl]ethenyl\}-1,1'-biphenyl (30e). To a suspension of 4,4'-bis[(triphenylphosphonio)methyl]-2-(ethylhexyloxy)biphenyl dibromide ( prepared according to the procedure as for compound 25$)(1 \mathrm{~g}, 1 \mathrm{mmol})$ in $20 \mathrm{~mL}$ of dry THF, $t$-BuOK $(0.28 \mathrm{~g}, 2.5 \mathrm{mmol})$ was added and the reaction mixture was stirred until complete dissolution phosphonium salt happened. Then a solution of $0.55 \quad \mathrm{~g}$ 4(diphenylamino)benzaldehyde $(2 \mathrm{mmol})$ in $10 \mathrm{~mL}$ of THF was slowly added dropwise. After $2 \mathrm{~h}$ stirring, the reaction mixture was poured into $100 \mathrm{ml}$ of water and extracted with chloroform. The combined organic solution was washed with brine, dried over $\mathrm{Na}_{2} \mathrm{SO}_{4}$ and then evaporated in vacuum. The residue was chromatographed on silica gel (chloroform) to yield 30e $(0.78 \mathrm{~g}$, $95 \%$ ) as white-yellow solid. After recryistallization from hexane. $\mathrm{MP}=76-77^{\circ} \mathrm{C}$; Elemental Anal. Calcd for $\mathrm{C}_{60} \mathrm{H}_{56} \mathrm{~N}_{2} \mathrm{O}$ : C 87.77, H 6.87, N 3.41 Found: C 88.06, H 6.99, N 3.54; ${ }^{1} \mathrm{H}$ NMR $\left(\mathrm{CDCl}_{3}, 500 \mathrm{MHz}\right), \delta: 0.87\left(\mathrm{t}, \mathrm{J}=7.0 \mathrm{~Hz}, 3 \mathrm{H},-\mathrm{CH}_{3}\right), 0.89\left(\mathrm{t}, 3 \mathrm{H}, \mathrm{J}=7.0 \mathrm{~Hz},-\mathrm{CH}_{3}\right), 1.2-1.5(\mathrm{~m}$, $\left.8 \mathrm{H},-\left(\mathrm{CH}_{2}\right)_{4}-\right), 1.70\left(\mathrm{~m}, 1 \mathrm{H},-\mathrm{OCH}_{2}-\mathrm{CH}=\right), 3.93\left(\mathrm{~d}, 2 \mathrm{H}, \mathrm{J}=5,5 \mathrm{~Hz},-\mathrm{O}-\mathrm{CH}_{2}-\right), 7.04$ (d, 2H, J = 16 $\mathrm{Hz}-\mathrm{CH}=\mathrm{CH}-), 7.0-7.2(\mathrm{~m}, 18 \mathrm{H}), 7.18\left(\mathrm{dd}, 1 \mathrm{H}, \mathrm{J}^{\text {ortho }}=8 \mathrm{~Hz}, \mathrm{~J}^{\text {meta }}=1.5 \mathrm{~Hz}, 5\right.$ biphenyl $), 7.29$ $(\mathrm{d}, 2 \mathrm{H}, \mathrm{J}=16 \mathrm{~Hz}-\mathrm{CH}=\mathrm{C} \underline{\mathrm{H}}-\mathrm{)}), 7.2-7.3(\mathrm{~m}, 7 \mathrm{H}), 7.37$ (d, 1H, J ${ }^{\text {ortho }}=8 \mathrm{~Hz}, 6$ biphenyl), 7.43 (d, $\left.4 \mathrm{H}, \mathrm{J}^{\text {ortho }}=9 \mathrm{~Hz}\right), 7.53\left(\mathrm{~d}, 2 \mathrm{H}, \mathrm{J}^{\text {ortho }}=9 \mathrm{~Hz}, 3^{\prime}, 5^{\prime}\right.$ biphenyl), $7.58\left(\mathrm{~d}, 2 \mathrm{H}, \mathrm{J}^{\text {ortho }}=9 \mathrm{~Hz}, 2^{\prime}, 6^{\prime}\right.$ biphenyl); IR (KBr, $\left.v \mathrm{~cm}^{-1}\right)$ : 2930, 2870, 1590, 1500, 1340, 1280, 1180, 970, 830, 760, 700. UV $\left(\mathrm{CHCl}_{3}, \lambda_{\max }, \mathrm{nm}\left(\varepsilon, 1 /\left(\mathrm{mol}^{*} \mathrm{~cm}\right)\right)\right): 382.0$ (40000); FLU $\left(\mathrm{CHCl}_{3}, \lambda_{\max }, \mathrm{nm}, \lambda_{\mathrm{EX}}=365.0 \mathrm{~nm}\right)$ 452.0.

2-Nitrobiphenyl-4, 4'-dicarboxylic acid dimethyl ester (31). To a solution of $20 \mathrm{~g}$ (74 mmol) of biphenyl-4, 4'-dicarboxylic acid dimethyl ester (1) in $200 \mathrm{ml}$ of conc. sulfuric acid was added $12 \mathrm{ml}(74.0 \mathrm{mmol})$ of $56 \% \mathrm{HNO}_{3}$ in $15 \mathrm{ml}$ of conc. $\mathrm{H}_{2} \mathrm{SO}_{4}$ dropwise at $15^{\circ} \mathrm{C}$ under intense stirring. The reaction mixture was maintained at $15-20^{\circ} \mathrm{C}$ for additional $1 \mathrm{~h}$ and then was carefully poured on a crushed ice. The precipitated solids were separated by filtration, washed with water, recrystallized from isopropanol and air-dried. Yield $20.2 \mathrm{~g}(86 \%)$ of the colorless crystals. $\mathrm{MP}=163-164^{\circ} \mathrm{C}$; Elemental Anal. Calcd for $\mathrm{C}_{16} \mathrm{H}_{13} \mathrm{NO}_{6}: \mathrm{C} 60.95, \mathrm{H} 4.16, \mathrm{~N} 4.44$ Found: C 60.56, H 4.06, N 4.32; ${ }^{1} \mathrm{H}$ NMR (DMSO-d $\left.{ }^{6}, 100 \mathrm{MHz}\right), \delta: 3.88$ (s, 3H, $\left.-\mathrm{CH}_{3}\right), 3.93$ (s, 
$\left.3 \mathrm{H},-\mathrm{CH}_{3}\right), 7.53\left(\mathrm{~d}, 2 \mathrm{H}, \mathrm{J}^{\text {ortho }}=8.6 \mathrm{~Hz}, 3^{\prime}, 5^{\prime} / 2^{\prime}, 6^{\prime}-\mathrm{Ar}\right), 7.73\left(\mathrm{~d}, 1 \mathrm{H}, \mathrm{J}^{\text {ortho }}=7.9 \mathrm{~Hz}, 6-\mathrm{Ar}\left(\mathrm{NO}_{2}\right)\right)$, $8.04\left(\mathrm{~d}, 2 \mathrm{H}, \mathrm{J}^{\text {ortho }}=8.6 \mathrm{~Hz}, 3^{\prime}, 5^{\prime} / 2^{\prime}, 6^{\prime}-\mathrm{Ar}\right), 8.28\left(\mathrm{dd}, 1 \mathrm{H}, \mathrm{J}^{\text {ortho }}=8.0 \mathrm{~Hz}, \mathrm{~J}^{\text {meta }}=1.7 \mathrm{~Hz}, 5-\right.$ $\left.\operatorname{Ar}\left(\mathrm{NO}_{2}\right)\right), 8.48\left(\mathrm{~d}, 1 \mathrm{H}, \mathrm{J}^{\text {meta }}=1.5 \mathrm{~Hz}, 3-\mathrm{Ar}\left(\mathrm{NO}_{2}\right)\right)$; IR $\left(\mathrm{KBr}, \mathrm{cm}^{-1}\right): 3107,3093,3027,2980$, 2900, 2863, 1733, 1620, 1543, 1443, 1373, 1323, 1293, 1253, 1217, 1200, 1173, 1147, 1133, 1123, 1040, 1023, 993, 980, 973, 940, 907, 890, 873, 837, 793, 787, 770, 720.

9H-Carbazole-2, 7-dicarboxylic acid dimethyl ester (32). A solution of $1.0 \mathrm{~g}$ (3.2 mmol) of 2azidobiphenyl-4, 4'-dicarboxylic acid dimethyl ester in $15 \mathrm{ml}$ of xylene was heated at reflux for $40 \mathrm{~h}$. When TLC indicated no starting material, the reaction mixture was cooled to RT, precipitate collected, recrystallized from xylene and air-dried. Yield $0.8 \mathrm{~g}$ (88\%), colorless crystals. $\mathrm{MP}=273-274^{\circ} \mathrm{C}$; Elemental Anal. Calcd for $\mathrm{C}_{16} \mathrm{H}_{13} \mathrm{NO}_{4}$ : C 67.84, H 4.63, N 4.94 Found: C 67.57, H 4.57, N 4.86; ${ }^{1} \mathrm{H}$ NMR (DMSO-d ${ }_{6}: \mathrm{CCl}_{4}(1: 1), 500 \mathrm{MHz}$ ), $\delta: 3.93$ (s, 6H, $\left.\mathrm{CH}_{3}\right), 7.80\left(\mathrm{dd}, 2 \mathrm{H}, \mathrm{J}^{\text {ortho }}=8.5 \mathrm{~Hz}, \mathrm{~J}^{\text {meta }}=1.3 \mathrm{~Hz}, 3,6\right.$-carbazolyl), 8.15 (s, 2H, 1,8-carbazolyl), $8.19\left(\mathrm{~d}, 2 \mathrm{H}, \mathrm{J}^{\text {ortho }}=8.3 \mathrm{~Hz}, 4,5\right.$-carbazolyl), $11,64(\mathrm{~s}, 1 \mathrm{H},-\mathrm{NH}-)$; IR $\left(\mathrm{KBr}, v_{\mathrm{cm}}{ }^{-1}\right): 3367,3083$, 2993, 2837, 2700, 2633, 2587, 2513, 1920, 1860, 1677, 1630, 1610, 1573, 1507, 1460, 1413, 1293, 1243, 1217, 1127, 1100, 1003, 943, 890, 880, 847, 827, 757, 723, 687, 657; UV (EtOH, $\left.\lambda_{\max }, \mathrm{nm}\right): 252.0,276.5,306.5,320.0$.

9(N)-Phenylcarbazole-2, 7-dicarboxylic acid dimethyl ester (33). To a solution of $285 \mathrm{mg}$ (1 mmol) of 9H-carbazole-2,7-dicarboxylic acid dimethyl ester (3) and $55 \mathrm{mg}(0.2 \mathrm{mmol})$ of dibenzo-18-crown-6 in $30 \mathrm{ml}$ of dry bromobenzene, $\mathrm{K}_{2} \mathrm{CO}_{3}(350 \mathrm{mg}, 2.5 \mathrm{mmol})$ and $\mathrm{CuI}(290$ $\mathrm{mg}, 1.5 \mathrm{mmol}$ ) was added and the reaction mixture was heated at reflux for $8 \mathrm{~h}$. After reaction finished (by TLC), inorganic solids were filtered off, the filtrate diluted with chloroform, washed with 5\% aq. hydrochloric acid and brine. The combined organic solution was concentrated in vacuum. The obtained beige crystals were recrystallized from benzene and air-dried. Yield 336 mg $(92 \%)$ colorless crystals. $\mathrm{MP}=210-211^{\circ} \mathrm{C}$; Elemental Anal. Calcd for $\mathrm{C}_{22} \mathrm{H}_{17} \mathrm{NO}_{4}$ : $\mathrm{C} 73.53$, H 4.77, N 3.90 Found: C 73.19, H 4.66, N 3.87; ${ }^{1} \mathrm{H}$ NMR (DMSO-d 6 , 500 MHz), $\delta: 3.85$ (s, 6H, $\left.-\mathrm{CH}_{3}\right), 7.67\left(\mathrm{~m}, 1 \mathrm{H}, \mathrm{J}^{\text {ortho }}=8.0 \mathrm{~Hz}, 4-\mathrm{Ph}\right), 7.68\left(\mathrm{dd}, 2 \mathrm{H}, \mathrm{J}^{\text {ortho }}=8.0 \mathrm{~Hz}, \mathrm{~J}^{\text {meta }}=1.0 \mathrm{~Hz}, 2-\mathrm{Ph}\right)$, $7.76\left(\mathrm{t}, 2 \mathrm{H}, \mathrm{J}^{\text {ortho }}=7.8 \mathrm{~Hz}, 3-\mathrm{Ph}\right), 7.89$ (s, 2H, 1,8-carbazolyl), 7.92 (dd, 2H, J ${ }^{\text {ortho }}=8.3 \mathrm{~Hz}, \mathrm{~J}^{\text {meta }}$ $=1.3 \mathrm{~Hz}, 3,6$-carbazolyl), 8.47 (d, $2 \mathrm{H}, \mathrm{J}^{\text {ortho }}=8.0 \mathrm{~Hz}, 4,5$-carbazolyl); IR $\left(\mathrm{KBr}, v \mathrm{~cm}^{-1}\right): 3428$, 3110, 3063, 3040, 3034, 2966, 2931, 2859, 1728, 1634, 1603, 1578, 1509, 1481, 1450, 1438, $1348,1303,1294,1253,1241,1225,1107,1009,894,828,763,737,716$; UV (EtOH, $\left.\lambda_{\max }, \mathrm{nm}\right)$ : $253.5,278.5,305.0,318.0$.

2,7-Bis(hydroxymethyl)-9(N)-phenylcarbazole. To a solution of $300 \mathrm{mg}(0,8 \mathrm{mmol})$ of 9(N)phenylcarbazole-2,7-dicarboxylic acid dimethyl ester (33) in $50 \mathrm{ml}$ of dry THF, $190 \mathrm{mg}$ (5 mmol) of $\mathrm{LiAlH}_{4}$ was added and the mixture was refluxed for $2 \mathrm{~h}$ under stirring. When TLC indicated the reaction was finished, the reaction mixture was treated with $10 \mathrm{ml}$ of ethyl acetate followed by $50 \%$ aq. solution of potassium hydroxide until aluminum hydroxide precipitated, which was filtered off and washed several times with hot THF. The organic phase was combined and solvent removed on the rotary evaporator. The crude product was recrystallized from ethanol. Yield $201 \mathrm{mg}(80 \%)$, light yellow powder. $\mathrm{MP}=211-213^{\circ} \mathrm{C}$; Elemental Anal. Calcd for $\mathrm{C}_{20} \mathrm{H}_{17} \mathrm{NO}_{2}$ : C 79.19, H 5.65, N 4.62 Found: C 78.89, H 5.18, N 4.56; ${ }^{1} \mathrm{H}$ NMR (DMSO-d 600 
MHz), $\delta: 4.62\left(\mathrm{~d}, 4 \mathrm{H}, \mathrm{J}=5.5 \mathrm{~Hz},-\mathrm{CH}_{2}-\right), 5.25(\mathrm{t}, 2 \mathrm{H}, \mathrm{J}=5.8 \mathrm{~Hz},-\mathrm{OH}), 7.20\left(\mathrm{dd}, 2 \mathrm{H}, \mathrm{J}^{\text {ortho }}=\right.$ $8.0 \mathrm{~Hz}, \mathrm{~J}^{\text {meta }}=0.5 \mathrm{~Hz}, 3,6$-carbazolyl), $7.33\left(\mathrm{~d}, 2 \mathrm{H}, \mathrm{J}^{\text {meta }}=0.5 \mathrm{~Hz}, 1,8\right.$-carbazolyl), 7.56 (m, 1H, $\left.\mathrm{J}^{\text {ortho }}=8.0 \mathrm{~Hz}, \mathrm{~J}^{\text {meta }}=1.3 \mathrm{~Hz}, 4-\mathrm{Ph}\right), 7.59\left(\mathrm{dd}, 2 \mathrm{H}, \mathrm{J}^{\text {ortho }}=8.5 \mathrm{~Hz}, \mathrm{~J}^{\text {meta }}=1.0 \mathrm{~Hz}, 2-\mathrm{Ph}\right), 7,71(\mathrm{t}$, $\left.2 \mathrm{H}, \mathrm{J}^{\text {ortho }}=7.8 \mathrm{~Hz}, 3-\mathrm{Ph}\right), 8.13\left(\mathrm{~d}, 2 \mathrm{H}, \mathrm{J}^{\text {ortho }}=8.0 \mathrm{~Hz}, 4,5\right.$-carbazolyl); IR $\left(\mathrm{KBr}, v \mathrm{~cm}^{-1}\right): 3343$, $3227,3110,3079,3043,3023,1603,1580,1503,1450,1436,1373,1340,1303,1240,1210$, $1177,1140,1043,1010,873,813,800,713$.

2,7-Diformyl-9(N)-phenylcarbazole (34). To a suspension of $150 \mathrm{mg}(0.5 \mathrm{mmol})$ of 2, 7bis(hydroxymethyl)-9(N)-phenylcarbazole in $30 \mathrm{ml}$ of dry dichloromethane, PCC in dioxane (500 $\mathrm{mg}$ of PCC and minimal amount of dioxane) was added at RT with intense stirring. The reaction was continued for $8 \mathrm{~h}$ and then $10 \mathrm{ml}$ of isopropanol was added. Inorganic solids were separated by flash chromatography, solvents removed in vacuum, the obtained brown solid was purified by column chromatography on silica gel ( dichloromethane). Yield $107 \mathrm{mg}$ (73\%), bright yellow solid. MP $\sim 196^{\circ} \mathrm{C}$ (subl.); Elemental Anal. Calcd for $\mathrm{C}_{20} \mathrm{H}_{13} \mathrm{NO}_{2}$ : C 80.25, $\mathrm{H} 4.38$, N 4.68 Found: C 79.89, H 4.17, N 4.61; ${ }^{1} \mathrm{H}$ NMR (DMSO-d $\left.6,500 \mathrm{MHz}\right), \delta: 7.65$ (m, 1H, J ${ }^{\text {ortho }}=$ $\left.7.3 \mathrm{~Hz}, \mathrm{~J}^{\text {meta }}=1.6 \mathrm{~Hz}, 4-\mathrm{Ph}\right), 7.73\left(\mathrm{dd}, 2 \mathrm{H}, \mathrm{J}^{\text {ortho }}=8.5 \mathrm{~Hz}, \mathrm{~J}^{\text {meta }}=1.5 \mathrm{~Hz}, 2-\mathrm{Ph}\right), 7.77\left(\mathrm{t}, 2 \mathrm{H}, \mathrm{J}^{\text {ortho }}\right.$ $=7.5 \mathrm{~Hz}, 3-\mathrm{Ph}), 7.88\left(\mathrm{dd}, 2 \mathrm{H}, \mathrm{J}^{\text {ortho }}=8.0 \mathrm{~Hz}, \mathrm{~J}^{\text {meta }}=1.0 \mathrm{~Hz}, 3,6\right.$-carbazolyl), 7.94 (s, 2H, 1,8carbazolyl), 8.57 (d, 2H, J ${ }^{\text {ortho }}=8.0 \mathrm{~Hz}, 4,5$-carbazolyl), 10.11 (s, 2H, -CHO); IR ( $\left.\mathrm{KBr}, \mathrm{v} \mathrm{cm}^{-1}\right)$ : 3083, 3043, 2973, 2943, 2853, 2777, 2760, 2710, 1687, 1623, 1597, 1507, 1473, 1447, 1433, $1407,1350,1297,1277,1240,1200,1170,1147,1100,1087,1043,1017,883,813,773,710$; UV (EtOH, $\left.\lambda_{\max }, \mathrm{nm}\right): 255.5,295.0,335.5$.

9(N)-Phenylcarbazole-2, 7-dicarboxylic acid dihydrazide (35). To a solution of $285 \mathrm{mg}$ (1 mmol) of 9(N)-phenylcarbazole-2, 7-dicarboxylic acid dimethyl ester (33) in $50 \mathrm{ml}$ of ethanol, $10 \mathrm{ml}$ (ca. $303 \mathrm{mmol}$ ) of hydrazine hydrate was added and the resulting mixture was refluxed for $3 \mathrm{~h}$. During that time a bulky precipitate was formed, which was separated from mother liquor, washed with ethanol and water, recrystallized from ethanol and air-dried. Yield $358 \mathrm{mg}$ (99\%). $\mathrm{MP} \sim 265^{\circ} \mathrm{C}$ (subl.); Elemental Anal. Calcd for $\mathrm{C}_{20} \mathrm{H}_{17} \mathrm{~N}_{5} \mathrm{O}_{2}$ : C 66.84, H 4.77, N 19.49 Found: C 66.53, H 4.68, N 19.40; ${ }^{1} \mathrm{H}$ NMR (DMSO-d $6,500 \mathrm{MHz}$ ), $\delta: 4.56$ (s, 4H, -NH ), 7.61 (м, 1H, $\left.\mathrm{J}^{\text {ortho }}=7.3 \mathrm{~Hz}, \mathrm{~J}^{\text {meta }}=1.4 \mathrm{~Hz}, 4-\mathrm{Ph}\right), 7.68\left(\mathrm{dd}, 2 \mathrm{H}, \mathrm{J}^{\text {ortho }}=9.0 \mathrm{~Hz}, \mathrm{~J}^{\text {meta }}=2.0 \mathrm{~Hz}, 2-\mathrm{Ph}\right), 7.74(\mathrm{t}$, $\left.2 \mathrm{H}, \mathrm{J}^{\text {ortho }}=7.8 \mathrm{~Hz}, 3-\mathrm{Ph}\right), 7.79\left(\mathrm{dd}, 2 \mathrm{H}, \mathrm{J}^{\text {ortho }}=8.0 \mathrm{~Hz}, \mathrm{~J}^{\text {meta }}=1.25 \mathrm{~Hz}, 3,6\right.$-carbazolyl), $7.86(\mathrm{~s}$, 2H, 1,8-carbazolyl), 8.36 (d, 2H, J ${ }^{\text {ortho }}=8.0 \mathrm{~Hz}, 4,5$-carbazolyl), 9.93 (s, 2H, -NH-); IR ( $\mathrm{KBr}, v$ $\mathrm{cm}^{-1}$ ): 3440, 3410, 3334, 3298, 3082, 2950, 2872, 1650, 1623, 1607, 1573, 1553, 1537, 1520, $1507,1467,1440,1360,1327,1273,1247,1177,1113,1047,1020,953,903,867,833,773$, 717.

2-Aminobiphenyl-4,4'-dicarboxylic acid dimethyl ester (36). To a solution of $14.6 \mathrm{~g}$ (46 mmol) of 2-nitrobiphenyl-4,4'-dicarboxylic acid dimethyl ester (31) in $500 \mathrm{ml}$ of dry THF, $10 \mathrm{~g}$ of $10 \% \mathrm{Pd} / \mathrm{C}$ was added and the reaction mixture was stirred up under hydrogen atmosphere at RT for 10 days. When the absorption of hydrogen was ceased, the catalyst was filtered off and solvents removed by the rotary evaporator. The resulting yellow paste was recrystallized from ethanol and dried under reduced pressure. Yield $12 \mathrm{~g} \mathrm{(91 \% )} \mathrm{of} \mathrm{light} \mathrm{yellow} \mathrm{to} \mathrm{white} \mathrm{solid.} \mathrm{MP} \mathrm{=}$ 160.0-160.5 ${ }^{\circ}$; Elemental Anal. Calcd for $\mathrm{C}_{16} \mathrm{H}_{15} \mathrm{NO}_{4}$ : C 67.36, H 5.30, N 4.91 Found: C 67.04, 
H 5.22, N 4.83; ${ }^{1} \mathrm{H}$ NMR (DMSO-d $\left.6,100 \mathrm{MHz}\right), \delta: 3.84$ (s, 3H, $\left.-\mathrm{CH}_{3}\right), 3.88\left(\mathrm{~s}, 3 \mathrm{H},-\mathrm{CH}_{3}\right), 5.16$ $\left(\mathrm{s}, 2 \mathrm{H},-\mathrm{NH}_{2}\right), 7.16\left(\mathrm{~s}, 1 \mathrm{H}, 3-\operatorname{Ar}\left(\mathrm{NH}_{2}\right)\right), 7.20\left(\mathrm{~d}, 1 \mathrm{H}, \mathrm{J}^{\mathrm{meta}}=1.3 \mathrm{~Hz}, 6-\operatorname{Ar}\left(\mathrm{NH}_{2}\right)\right), 7.45(\mathrm{~d}, 1 \mathrm{H}$, $\left.\mathrm{J}^{\text {meta }}=1.0 \mathrm{~Hz}, 5-\operatorname{Ar}\left(\mathrm{NH}_{2}\right)\right), 7.59\left(\mathrm{~d}, 2 \mathrm{H}, \mathrm{J}^{\text {ortho }}=8.5 \mathrm{~Hz}, 3^{\prime}, 5^{\prime} / 2^{\prime}, 6^{\prime}-\mathrm{Ar}\right), 8.03\left(\mathrm{~d}, 2 \mathrm{H}, \mathrm{J}^{\text {ortho }}=8.5\right.$ $\left.\mathrm{Hz}, 3^{\prime}, 5^{\prime} / 2^{\prime}, 6^{\prime}-\mathrm{Ar}\right)$; IR (KBr, $\left.v \mathrm{~cm}^{-1}\right)$ : 3475, 3380, 3225, 3100, 3070, 3047, 3015, 2960, 2900, 2845, 1943, 1913, 1807, 1780, 1720, 1630, 1610, 1570, 1560, 1530, 1490, 1440, 1403, 1367 , $1340,1313,1303,1283,1267,1250,1190,1157,1123,1113,1073,1020,1003,970,953,910$, $867,840,827,793,773,760,727,700$.

2-Azidobiphenyl-4,4'-dicarboxylic acid dimethyl ester (37). To a $2.9 \mathrm{~g}$ (10.1 mmol) of 2aminobiphenyl-4,4'-dicarboxylic acid dimethyl ester 36, the mixture of $40 \mathrm{ml}$ of hydrochloric acid and $20 \mathrm{ml}$ of water was added and the resulted solution was stirred at RT for $1 \mathrm{~h}$. The obtained suspension then was cooled to $0^{\circ} \mathrm{C}$ and the solution of $0.7 \mathrm{~g}(10.2 \mathrm{mmol})$ of $\mathrm{NaNO}_{2}$ in minimal amount of water was added dropwise within $1 \mathrm{~h}$ at $0-5^{\circ} \mathrm{C}$ and constant stirring. After the reaction mixture was stirred at $0^{\circ} \mathrm{C}$ for additional $1 \mathrm{~h}$, the solution of $0.65 \mathrm{~g}(10 \mathrm{mmol})$ of $\mathrm{NaN}_{3}$ in minimal amount of water was added dropwise under intense stirring, and the reaction continued at RT for another $2 \mathrm{~h}$. The precipitated solid was filtered off, washed with water and dried under reduced pressure in the dark. Yield $3.1 \mathrm{~g}(99 \%)$, light-yellow or white powder. $\mathrm{MP}=$ 107-108 ${ }^{\circ} \mathrm{C}$ (dec.); Elemental Anal. Calcd for $\mathrm{C}_{16} \mathrm{H}_{13} \mathrm{~N}_{3} \mathrm{O}_{4}$ : C 61.73, H 4.21, N 13.50 Found: C 61.45, H 4.13, N 13.39; ${ }^{1} \mathrm{H}$ NMR (DMSO-d $6,100 \mathrm{MHz}$ ), $\delta: 3.93$ (s, 3H, $-\mathrm{CH}_{3}$ ), 3.95 (s, 3H, $\left.\mathrm{CH}_{3}\right), 7.43\left(\mathrm{~s}, 1 \mathrm{H}, \operatorname{Ar}\left(-\mathrm{N}_{3}\right)\right), 7.47\left(\mathrm{~s}, 1 \mathrm{H}, \operatorname{Ar}\left(-\mathrm{N}_{3}\right)\right), 7.56\left(\mathrm{~s}, 1 \mathrm{H}, \operatorname{Ar}\left(-\mathrm{N}_{3}\right)\right), 7.87\left(\mathrm{~d}, 2 \mathrm{H}, \mathrm{J}^{\text {ortho }}=8.4\right.$ $\left.\mathrm{Hz}, 3^{\prime}, 5^{\prime} / 2^{\prime}, 6^{\prime}-\mathrm{Ar}\right), 8.09$ (d, 2H, J' $\left.{ }^{\text {ortho }}=8.2 \mathrm{~Hz}, 3^{\prime}, 5^{\prime} / 2^{\prime}, 6^{\prime}-\mathrm{Ar}\right)$; IR $\left(\mathrm{KBr}, v^{-1}\right)$ : 3100, 3077 , 3050, 3013, 2960, 2857, 2160, 2120, 1950, 1723, 1713, 1607, 1573, 1560, 1557, 1517, 1493, $1437,1417,1397,1377,1337,1290,1283,1270,1260,1250,1203,1197,1160,1123,1027$, 1010, 993, 977, 963, 890, 873, 850, 833, 807, 780, 757, 733, 707, 683, 653.

Dimethyl 2-iodobiphenyl-4, 4'-dicarboxylate (38). Dimethyl 2-aminobiphenyl-4,4'dicarboxylate (36) $1.42 \mathrm{~g}$ (5 mmol) was suspended in $5 \mathrm{ml}$ of $15 \%$ hydrochloric acid, the suspension was cooled at $0^{\circ} \mathrm{C}$ and a solution of $\mathrm{NaNO}_{2}(0.4 \mathrm{~g}, 5.8 \mathrm{~mol})$ in $3 \mathrm{ml}$ of water was added dropwise. The mixture was stirred at $0^{\circ} \mathrm{C}$ for $10 \mathrm{~min}$, then $0.9 \mathrm{~g}(6 \mathrm{mmol}) \mathrm{NaI}$ was added and the resulting solution was heated to $60^{\circ} \mathrm{C}$ for $10 \mathrm{~min}$. The reaction mixture was poured into $50 \mathrm{ml}$ of water and extracted with ethyl acetate. The combined organic solution was washed with $10 \%$ solution of sodium sulfite and then was dried over $\mathrm{Na}_{2} \mathrm{SO}_{4}$. After removal of solvent, a residue was purified by column chromatography on silica gel (toluene) to give 38 (1.2 g, 60\%) as colorless solid. Recrystallization from ethanol afforded white needle-like crystals. $\mathrm{MP}=112$ $113^{\circ} \mathrm{C}$; Elemental Anal. Calcd for $\mathrm{C}_{16} \mathrm{H}_{13} \mathrm{IO}_{4}$ : C 48.51, H 3.31 Found: C 48.01, H 3.30; ${ }^{1} \mathrm{H}$ NMR $\left(\mathrm{CDCl}_{3}, 500 \mathrm{MHz}\right), \delta: 3.89\left(\mathrm{~s}, 6 \mathrm{H},-\mathrm{CH}_{3}\right), 7.29\left(\mathrm{~d}, 1 \mathrm{H}, \mathrm{J}^{\text {ortho }}=8.0 \mathrm{~Hz}, 6\right.$ biphenyl ), $7.36(\mathrm{~d}, 2 \mathrm{H}$, $\mathrm{J}^{\text {ortho }}=8.5 \mathrm{~Hz}, 2^{\prime}, 6^{\prime}$ ' or 3', 5' biphenyl), $7.99\left(\mathrm{dd}, 1 \mathrm{H}, \mathrm{J}^{\text {ortho }}=8.0 \mathrm{~Hz}, \mathrm{~J}^{\text {meta }}=1.5 \mathrm{~Hz}, 5\right.$ biphenyl $)$, $8.06\left(\mathrm{~d}, 2 \mathrm{H}, \mathrm{J}^{\text {ortho }}=8.5 \mathrm{~Hz}, 3^{\prime}, 5^{\prime}\right.$ or 2', 6' biphenyl $), 8.56\left(\mathrm{~d}, 1 \mathrm{H}, \mathrm{J}^{\text {meta }}=1.5 \mathrm{~Hz}, 3 \mathrm{biphenyl}\right)$; IR $\left(\mathrm{KBr}, v \mathrm{~cm}^{-1}\right): 2970,1730,1440,1290,1250,1140,970,850,770,720$.

2, 7-Bis [2-(benzoxazol-2-yl)-(E)-ethenyl]-9(N)-phenylcarbazole (39). A solution of $100 \mathrm{mg}$ (0.3 mmol) of 2,7-diformyl-9(N)-phenylcarbazole (34) in $15 \mathrm{ml}$ of dry DMSO was treated with $110 \mathrm{mg}(0.8 \mathrm{mmol})$ of 2-methyl-1,3-benzoxazole in the presence of $111 \mathrm{mg}(1 \mathrm{mmol}) \mathrm{t}$-BuOK . 
Reaction mixture was heated at $40-50^{\circ} \mathrm{C}$ for $16 \mathrm{~h}$ under stirring, and then was diluted with brine and extracted with chloroform. Organic phases were combined and solvents removed in vacuum. The residue was purified by column chromatography on silica gel (chloroform) and dried under reduced pressure. Recrystallization from benzene gave 39 (138 mg, 78\%), as bright green-yellow crystals. $\mathrm{MP}=306-309^{\circ} \mathrm{C}$ (dec.); Elemental Anal. Calcd for $\mathrm{C}_{36} \mathrm{H}_{23} \mathrm{~N}_{3} \mathrm{O}_{2}: \mathrm{C} 81.65, \mathrm{H} 4.38, \mathrm{~N}$ 7.93 Found: C 81.84, H 4.13, N 7.71; ${ }^{1} \mathrm{H}$ NMR (DMSO-d $\left.6,500 \mathrm{MHz}\right), \delta: 7.32-7.45$ (m, 4H, 5',5",6',6"-oxazolyl), $7.63\left(\mathrm{~m}, 1 \mathrm{H}, \mathrm{J}^{\text {ortho }}=6.5 \mathrm{~Hz}, \mathrm{~J}^{\text {meta }}=1.3 \mathrm{~Hz}, 4-\mathrm{Ph}\right), 7.65-7.80(\mathrm{~m}, 8 \mathrm{H}$, 4',4", 7',7"'-oxazolyl + 2,3-Ph), $7.77(\mathrm{~d}, 2 \mathrm{H}, \mathrm{J}=16.0 \mathrm{~Hz},=\mathrm{CH}-), 7.86\left(\mathrm{dd}, 2 \mathrm{H}, \mathrm{J}^{\text {ortho }}=8.0 \mathrm{~Hz}\right.$, $\mathrm{J}^{\text {meta }}=1.0 \mathrm{~Hz}, 3,6$-carbazolyl), $8.00(\mathrm{~d}, 2 \mathrm{H}, \mathrm{J}=16.0 \mathrm{~Hz},=\mathrm{CH}-), 8.31$ (s, 2H, 1,8-carbazolyl), 8.37 (d, 2H, J ${ }^{\text {ortho }}=8.0 \mathrm{~Hz}, 4,5$-carbazolyl); IR $\left(\mathrm{KBr}, \mathrm{v} \mathrm{cm}^{-1}\right): 3072,3039,2971,2941,2872$, $1639,1600,1571,1534,1505,1456,1430,1354,1321,1305,1292,1250,1233,1207,1184$, $1171,1154,1118,1085,1039,1017,975,944,905,885,869,846,833,807,770,754,708$; UV (DMF, $\left.\lambda_{\max }, \mathrm{nm}\left(\varepsilon, 1 /\left(\mathrm{mol}^{*} \mathrm{~cm}\right)\right)\right): 386.0$ (71300), 408.5 (shoulder, 56000); FLU (DMF, $\lambda_{\max }, \mathrm{nm}$, $\left.\lambda_{\mathrm{EX}}=365.0 \mathrm{~nm}\right) 457.5$.

2, 7-Bis [5-(4-bromophenyl)-1,3,4-oxadiazol-2-yl]-N-phenylcarbazole (40). A solution of 9(N)-phenylcarbazole-2,7-dicarboxylic acid dihydrazide 35 (300 mg, 0,8 mmol) in $30 \mathrm{ml}$ of dry pyridine was treated with 4-bromobenzoyl chloride $(455 \mathrm{mg}, 2,1 \mathrm{mmol})$ at $60^{\circ} \mathrm{C}$ for $16 \mathrm{~h}$ under stirring. Then the reaction mixture was diluted with water, a precipitate was collected and washed with water. The obtained substance was recrystallized from benzene and dried under reduced pressure.

The crude product was placed in $30 \mathrm{ml}$ of $\mathrm{POCl}_{3}$ and heated at reflux for $8 \mathrm{~h}$. The reaction mixture was poured on crushed ice, the precipitated product was filtered off, washed with the sat. solution of sodium bicarbonate and water. The crude product was placed into the Soxhlet apparatus to remove the contaminants with cyclohexane, and then a residue was purified by column chromatography on silica gel (dichloromethane) and, finally, recrystallized from dimethyl sulfoxide. Yield (2 steps) $262 \mathrm{mg}(46 \%)$. MP $\sim 350^{\circ} \mathrm{C}$ (subl.); Elemental Anal. Calcd for $\mathrm{C}_{34} \mathrm{H}_{19} \mathrm{Br}_{2} \mathrm{~N}_{5} \mathrm{O}_{2}$ : C 59.24, H 2.78, Br 23.18, N 10.16 Found: C 58.91, H 2.54, Br 23.26, N 10.28; ${ }^{1} \mathrm{H}$ NMR (DMSO-d $\left.6,500 \mathrm{MHz}\right), \delta: 7.71\left(\mathrm{~m}, 1 \mathrm{H}, \mathrm{J}^{\text {ortho }}=6.5 \mathrm{~Hz}, \mathrm{~J}^{\text {meta }}=1.3 \mathrm{~Hz}, 4-\mathrm{Ph}\right), 7.79$ $\left(\mathrm{dd}, 1 \mathrm{H}, \mathrm{J}^{\text {ortho }}=7.0 \mathrm{~Hz}, \mathrm{~J}^{\text {meta }}=1.3 \mathrm{~Hz}, 2-\mathrm{Ph}\right), 7.80-7.88\left(\mathrm{~m}, 6 \mathrm{H}, 3^{\prime}-(\mathrm{Br}) \mathrm{Ph}+3-\mathrm{Ph}\right), 8.00-8.08$ $\left(\mathrm{m}, 4 \mathrm{H}, 2^{\prime}-(\mathrm{Br}) \mathrm{Ph}\right), 8.14\left(\mathrm{dd}, 2 \mathrm{H}, \mathrm{J}^{\text {ortho }}=8.0 \mathrm{~Hz}, \mathrm{~J}^{\text {meta }}=1.0 \mathrm{~Hz}, 3,6\right.$-carbazolyl), 8.47 (s, 2H, 1,8carbazolyl), 8.62 (d, $2 \mathrm{H}, \mathrm{J}^{\text {ortho }}=8.0 \mathrm{~Hz}, 4,5$-carbazolyl); IR $\left(\mathrm{KBr}, \mathrm{v} \mathrm{cm}^{-1}\right): 3100,3083,2937$, 2870, 1687, 1603, 1580, 1547, 1477, 1413, 1400, 1370, 1360, 1347, 1310, 1283, 1273, 1247, 1237, 1193, 1183, 1130, 1120, 1086, 1017, 973, 847, 747, 720; UV (DMF, $\lambda_{\max }, \mathrm{nm}(\varepsilon$, 1/(mol*cm))): $340.0(87300)$; FLU (DMF, $\left.\lambda_{\max }, \mathrm{nm}, \lambda_{\mathrm{EX}}=365.0 \mathrm{~nm}\right) 428.5$.

2,7-Bis\{5-[4-(octyloxy)phenyl]-1,3,4-oxadiazol-2-yl\}-9,10-diphenylphenanthrene (41). To a solution of $0.21 \mathrm{~g}(0.47 \mathrm{mmol})$ dimethyl 9, 10-diphenylphenanthrene-2, 7-dicarboxylate (5) in 25 $\mathrm{ml}$ of THF, $10 \mathrm{ml}$ of $10 \%$ ethanol solution of $\mathrm{NaOH}$ was added. The mixture was refluxed for 3 h. After cooling at RT, $5 \mathrm{ml}$ of $20 \%$ hydrochloric acid was added to give a precipitate. The precipitate was filtered off and, after drying in a vacuum desiccator, was dissolved in $5 \mathrm{ml}$ of $\mathrm{SOCl}_{2}$. A drop of DMF was added and the mixture was refluxed for $2 \mathrm{~h}$. After the removal of 
excess of a $\mathrm{SOCl}_{2}$ in vacuum, the residue was dissolved in $25 \mathrm{ml}$ of THF and added dropwise to a solution of $0.26 \mathrm{~g}$ (1 mmol) 4-(octyloxy)benzohydrazide and $0.1 \mathrm{~g}(1 \mathrm{mmol})$ triethylamine in 5 $\mathrm{ml}$ of THF. The reaction mixture was stirred for $3 \mathrm{~h}$ at room temperature and then was poured into $50 \mathrm{ml}$ of water. The precipitate was collected by filtration and washed with water. After drying, white powder was dissolve in $5 \mathrm{ml}$ of $\mathrm{POCl}_{3}$ and the mixture was refluxed for $5 \mathrm{~h}$. The reaction mixture was poured on $100 \mathrm{ml}$ of crushed ice and extracted with chloroform. The combined organic phase was washed with water and then dried over $\mathrm{Na}_{2} \mathrm{SO}_{4}$. After removal of solvents, the residue was purified by column chromatography on silica gel (toluene/ ethyl acetate = 2: 1) to give $41(270 \mathrm{mg}, 66 \%)$ as a colorless solid. Recrystallization from hexane afforded white crystals. $\mathrm{MP}=163-165^{\circ} \mathrm{C}$; Elemental Anal. Calcd for $\mathrm{C}_{58} \mathrm{H}_{58} \mathrm{~N}_{4} \mathrm{O}_{4}: \mathrm{C} 79.60, \mathrm{H} \mathrm{6.68}, \mathrm{N}$ 6.40 Found: C79.27, H 6.56, N 6.32; ${ }^{1} \mathrm{H}$ NMR $\left(\mathrm{CDCl}_{3}, 500 \mathrm{MHz}\right) \delta: 0.90\left(\mathrm{t}, 6 \mathrm{H}, \mathrm{J}^{\text {ortho }}=7.0 \mathrm{~Hz}\right.$, $\left.-\mathrm{CH}_{3}\right), 1.2-1.7\left(\mathrm{~m}, 24 \mathrm{H},-\left(\mathrm{CH}_{2}\right)_{6}-\right), 3.98$ (t, 4H, J= 7.0 Hz, -O- $\left.\mathrm{CH}_{2-}\right), 6.9-8.9$ (m, 24H, arom.); ${ }^{13} \mathrm{C} \mathrm{NMR}\left(\mathrm{CDCl}_{3}, 125.75 \mathrm{MHz}\right) \delta: 14.1,22.7,25.9,29.3,29.7,31.8,68.8,114.9,117.7,122.9$, $123.5,124.6,126.3,126.6,127.7,127.9,128.1,129.3,129.5,130.8,136.4,157.4,164.5$; IR $\left(\mathrm{KBr}, v \mathrm{~cm}^{-1}\right): 3450,2930,2860,1710,1610,1490,1300,1250,1170,1070,1030,1000,840$, 740, 700; UV $\left(\mathrm{CHCl}_{3}, \lambda_{\max }, \mathrm{nm}\left(\varepsilon, 1 /\left(\mathrm{mol}^{*} \mathrm{~cm}\right)\right)\right): 288.0$ (26400); 335 (28800), FLU $\left(\mathrm{CHCl}_{3}\right.$, $\left.\lambda_{\text {max }}, \mathrm{nm}, \lambda_{\mathrm{EX}}=365.0 \mathrm{~nm}\right) 404.5,423.5$.

\section{Acknowledgements}

This work was carried out as a part of the research project of the State Program of Focused Fundamental Research, State registration No 2063025 and State Program of Applied Research, State registration N 2062331.

\section{References and Notes}

1. (a) Torssell, K. G. B. Natural Product Chemistry, Wiley: Chichester, 1983. (b) Manitto, P. Biosynthesis of Natural Products, Ellis Horwood: Chichester, 1981. (c) Thomson, R. H. The Chemistry of Natural Products, Blackie and Son: Glasgow, 1985. (d) Cordell, G. A. Introduction to Alkoloids, A Biogenelic Approach, Wiley: New York, 1981. (e) Ehrenfreund, J.; Lamberth, C.; Tobler, H.; Walter, H. WO Patent 2004/058723; Chem. Abstr. 2005, 141, 123619. (f) Herradon, G. B.; Alonso, G. M.; Benito, C. E.; Chana, L. A. Patent WO 2005/108354; Chem. Abstr. 2006, 143, 478203.

2. (a) Cram, D. J. Angew. Chem. 1988, 100, 1041. (b) Cram, D.J. Angew. Chem. Int. Ed. 1988, 27, 1009.

3. Mikes, F.; Boshart, G. J. Chromatogr. 1978, 149, 455.

4. (a) Brandmeier, V.; Feigel, M.; Bremer, M. Angew. Chem. 1989, 101, 466. (b) Brandmeier, V.; Feigel, M.; Bremer, M. Angew. Chem. Int. Ed. 1989, 28, 486. 
5. Tabushi, I.; Yamamura, K.; Nabeshima, T. J. Am. Chem. Soc. 1984, 106, 5267.

6. (a) Yamamura, K.; Ono, S.; Tabushi, I. Tetrahedron Lett. 1988, 29, 1797. (b) Yamamuro, K.; Ono, S.; Ogoshi, H.; Masuda, H.; Kuroda, Y. Synlett 1989, 18.

7. (a) Wincler, B.; Meghdadi, F.; Tasch, S.; Evers, B.; Schneider, I.; Fischer, W.; Stelzer, F.; Leising, G. Synthetic Metals 1999, 102, 1083. (b) Hohnholz, D.; Schweikart, K.-H.; Subramanian, L. R.; Wedel, A.; Wischert, W.; Hanack, M. Synthetic Metals 2000, 110, 141.

8. (a) Yao, T.; Campo, M. A.; Larock, R. C. J. Org. Chem. 2005, 70, 3511. (b) Freeman, A. W.; Urvoy, M.; Criswell, M. E. J. Org. Chem. 2005, 70, 5014.

9. (a) Razina, S. L.; Gricev, A. M.; Olkhovik V. K.; Yukhimets, N. V. Patent BY N $8072(6$ June 2006), 2006. (b) Olkhovik, V. K.; Matveyenko, Y. V.; Kalechyts, G. V.; Pap, A. A.; Zeniuk, A. A.; Erdman, A. A. Patent BY N 5137 (21 January 2003), 2003.

10. Olkhovik, V. K.; Matveyenko, Y. V.; Kalechyts, G. V.; Pap, A. A.; Zeniuk, A. A.; Erdman, A. A. Patent BY N 6194 (30 January 2004), 2004.

11. Olkhovik, V. K.; Matveyenko, Y. V.; Kalechyts, G. V.; Pap, A. A.; Zeniuk, A. A. Russ. J. Org. Chem. 2006, 42, 1164.

12. Youngeup Jin, Jinwoo Kim, Sung Heum Park, Kwanghee Lee, Hongsuk Suh. Bull. Korean Chem. Soc. 2005, 26, 795.

13. (a) Son, S.-H.; Yun, J.-J.; Oh, G.-Ch.; Jung, S.-Y.; Kim, Y.-K.; Kuhta, A.V.; Olkhovik, V. K.; Sasnouski, G.; Han, E.-M. Current Applied Physics 2005, 5, 75. (b) Kukhta, V. A.; Kolesnik, E. E.; Dudko, E. V.; Olkhovik, V. K.; Galinovskii, N. A.; Agabekov, V. E.; Kazakov, S. M.; Ivanov, A. L. J. Applied Spectroscopy 2007, 74, 684.

14. Hohloch, M.; Segura, J. L.; Döttinger, S. E.; Hohnholz, D.; Steinhuber, E.; Spreitzer, H.; Hanack M. Synth. Metals 1997, 84, 319.

15. Grigalevicius, S.; Lideikis, E.; Grazulevicius, J. V.; Gaidelis, V.; Antulis, J.; Jankauskas, V.; Tran Van, F.; Chevrot, C. Polymer 2002, 43, 5693.

16. Chia-Hua Lin, Ya-Ting Chang, Chen-Shun Li, Charng-Hsing Liu, Jiun-Pey Duan. Synth. Metals 2006, 156, 671.

17. Ciszek, J. W.; Tour, J. M. Tetrahedron Lett. 2004, 45, 2801.

18. Zheng, Sh.; Shi, J.; Mateu, R. Chem. Mater. 2000, 12, 1814.

19. Schlick, H.; Stelzer, F.; Leising, G. Monatsch. Chem. 2001, 132, 441.

20. Campaingne E.; Archer W. L. Org. Synth. Coll. 1963, 4, 331.

21. O’Brien, D. F.; Burrows, P. E.; Forrest, S. R.; Koene, B. E.; Loy, D. E.; Thompson, M. E. Adv. Mater. 1998, 10, 1108.

22. Guey-Sheng Liou, Sheng-Huei Hsiao, Hwei-Wen Chen. J. Mater. Chem. 2006, 16, 1831.

23. Cadogan, J. I. G. Synthesis 1969, 11.

24. Larock, R. C.; Doty, M. J.; Tian, Q.; Zenner, J. M. J. Org. Chem. 1997, 62, 7536.

25. Mandal, A. B.; Lee, G.-H.; Liu, Yi-H.; Peng, Sh.-M.; Leung, M. J. Org. Chem. 2000, 65, 332.

26. Janietz, S.; Anlauf, S.; Wedel, A. Synth. Metals 2001, 122, 11. 